\title{
金催化炔基膦酸酯水合反应合成 $\beta$-羰基膦酸酯
}

\author{
刘开建* 刘宏伟＼cjkstart王文革＼cjkstart欧丽娟＼cjkstart王津津＼cjkstart胡波年*
}

(湖南工学院材料与化学工程系 衡阳 421002)

\begin{abstract}
摘要 $\beta$-羰基膦酸酯是一类重要的有机化合物和反应中间体，在有机合成及药物化学中发挥着重要的作用. 提供了一 种高原子经济性、高选择性、温和的炔基膦酸酯水合反应体系. 实验结果表明: 在阳离子金催化剂(2.5 mol\%)的催化作 用下，以 1,2-二氯乙烷 $(1 \mathrm{~mL})$ 为溶剂，室温下炔基膦酸酯 $(1 \mathrm{mmol})$ 与水 $(3 \mathrm{mmol})$ 发生水合反应，高收率、高区域选择性地 得到 $\beta$-羰基膦酸酯化合物(收率 $\geqslant 92 \%$ ). 该方法具有底物适用范围广、反应条件温和、环境友好等优点, 为含 $\beta$-羰基膦 酸酯结构单元的天然产物及复杂药物分子的合成提供了一种新途径.
\end{abstract}

关键词＼cjkstart炔基膦酸酯; $\beta$-羰基膦酸酯化合物; 金催化; 水合反应

\section{Effective Transformation of Alkynylphosphonates into $\beta$-Ketophosphonates with a Gold(I) Catalyst}

\author{
Liu, Kaijian* Liu, Hongwei Wang, Wenge Ou, Lijuan Wang, Jingjing \\ $\mathrm{Hu}$, Bonian* \\ (Department of Materials and Chemical Engineering, Hunan Institute of Technology, Hengyang 421002)
}

\begin{abstract}
Ketophosphonates are an important class of carbonyl compounds which exhibit a wide range of biological activities and outstanding metal-complexing abilities. They are building blocks in organic synthesis, especially as intermediates for the synthesis of $\alpha, \beta$-unsaturated carbonyl compounds, chrial $\beta$-hydroxy and $\beta$-hydroxy phosphonic acids. In this paper, a simple, convenient and environmentally benign method to synthesize the $\beta$-ketophosphonates from alkynylphosphonates was developed, which can be easily prepared in one step from commercial terminal alkynes. In the presence of $2.5 \mathrm{~mol} \%$ $\mathrm{XPhosAuSbF}_{6}$ and 3 equiv. of water in dichloroethane at room temperature, a broad range of alkynylphosphonates were converted into the corresponding $\beta$-ketophosphonates in nearly equivalent conversion through gold-catalyzed hydration reaction. The presented methodology will provide new strategies for $\beta$-ketophosphonates-type natural product and drug synthesis, which has important academic significance and application value.
\end{abstract}

Keywords alkynylphosphonates; $\beta$-ketophosphonate; gold catalysis; hydration reaction

炔烃水合反应是指水分子与过渡金属或酸活化的 炔基化合物发生加成反应生成羰基化合物 ${ }^{[1,2]}$, 该反应 具有原子经济性好和环境友好的特点, 自从 1881 年被 Kucherov 首次发现以来就吸引了化学工作者的关注 ${ }^{[3]}$. $\mathrm{c}^{[4,5]}$ 、铂 ${ }^{[6,7]}$ 、铁 ${ }^{[8 \sim 10]}$ 、银 ${ }^{[11 \sim 13]}$ 、钴 ${ }^{[14]}$ 、硫酸 ${ }^{[15]}$ 等 ${ }^{[16]}$ 都被用于催化炔烃水合反应. 近年来, 均相金催化 反应由于具有反应条件温和、实验操作简便、官能团兼 容性好等优点吸引了越来越多的关注 ${ }^{[17 \sim 21]}$, 其中金催 化炔烃水合反应发展迅速 ${ }^{[22 ~ 31]}$. 但是目前金催化水合
反应的底物主要集中于末端炔烃、二烷(芳)基炔烃、烷 基芳基炔烃，关于杂原子取代的炔烃相关研究还较少， 因此非常有必要探索金催化杂原子取代炔烃的水合反 应，发展 $\alpha$-取代羰基化合物的新合成方法.

$\beta$-羰基膦酸酯是一种非常有价值的羰基化合物，作 为重要的合成中间体广泛应用于有机合成 ${ }^{[32 ~ 34]}$, 特别 是用于 Horner-Wadsworth-Emmons (HWE) 反应来合成

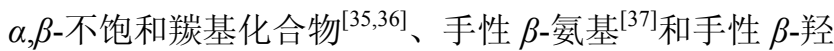
基 ${ }^{[38,39]}$ 膦酸化合物. 此外, $\beta$-羰基膦酸酯具有多种生物

*E-mail: liukaijian_1982@163.com; hbonian@163.com

Received April 24, 2014; revised September 3, 2014; published online September 9, 2014.

Project supported by the Science and Technology Planning Project of Hunan Province (No. 2014FJ3053), the National Natural Science Foundation of China (No. 21176061), the Natural Science Foundation of Hunan Province (No. 13JJ3132), the Construct Program of the Key Discipline in Hunan Province and Science and Technology Planning Project of Hengyang City (No. 2014FJ3053).

湖南省科技计划(No. 2014FJ3053)、国家自然科学基金(No. 21176061)、湖南省自然科学基金(No. 13JJ3132)、湖南省重点建设学科和衡阳市科技计划 资助项目. 
活性, 大量存在于天然产物和药物分子中. 目前, 已有 多种 $\beta$-羰基膦酸酯的合成方法 ${ }^{[40 ~ 44]}$, 特别是厦门大学 赵玉芬教授课题组 ${ }^{[40]}$ 发展了一种 $\mathrm{Pd}$ 催化的炔基膦酸酯 水合反应方法，该方法对 $\beta$-羰基膦酸酯的合成具有收率 高、普适性好等优点, 具有十分重要的参考价值. 但是 发展另外一种高原子经济性, 特别是反应条件温和, 官 能团兼容性好的合成方法也是十分有必要的. 实验结果 表明，在催化量的阳离子金催化剂作用下，炔基膦酸酯 ${ }^{[45]}$ 可以高选择性高产率的生成 $\beta$-羰基膦酸酯.

\section{1 结果与讨论}

\section{1 金催化炔基膦酸酯水合反应条件优化}

2-苯乙炔基膦酸二异丙酯(1a) (1.0 mmol), 水(3.0

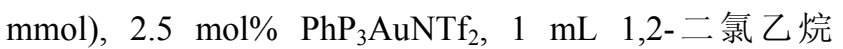
(DCE), 用 TLC 监控反应，室温搅拌反应 $24 \mathrm{~h}$ 后，仍有 部分原料末反应，通过核磁氢谱分析反应体系发现底物 $1 \mathrm{a}$ 的转化率仅 76\%, 相应的水合产物(2-苯乙基-2-氧代) 膦酸二异丙酯 $(\mathbf{2 a})$ 核磁产率为 74\% (Table 1, Entry 1). 用 上述投料比, 以 2-苯乙炔基膦酸二异丙酯为模型底物, 进行反应条件的优化, 2-苯乙炔基膦酸二异丙酯在不同 条件下的水合反应结果见表 1.

首先研究金催化剂配体的电子性质对反应的影响, 研究发现金催化剂配体的电子性质对炔基膦酸酯的转 化率影响非常小. 发现无论是含富电子的(4-MeO$\left.\mathrm{C}_{6} \mathrm{H}_{4}\right)_{3} \mathrm{P}$ 配体、富电子氮杂环配体 $[1,3$-双(2,6-二异丙基 苯基)咪唑-2-亚基](IPr), 还是含缺电子的 $\left(4-\mathrm{CF}_{3} \mathrm{C}_{6} \mathrm{H}_{4}\right)_{3} \mathrm{P}$ 配体在金催化剂作用下，反应的转化率基本不变 (Entries 2 4). 金催化剂配体体积对水合反应转化率的 实验结果表明, 催化剂的体积因素在炔基膦酸酯的水合 反应中发挥着重要的作用, 底物的转化率随着金催化剂 所使用的配体体积的增大呈上升趋势 (Scheme 1) (Entries $5 \sim 10$, BrettPhos $>$ Xphos $>$ SPhos $>$ MePhos $>$ L $>$ $\left.\mathrm{Ph}_{3} \mathrm{P}>\mathrm{Cy}_{3}>\mathrm{Et}_{3} \mathrm{P}\right)$.

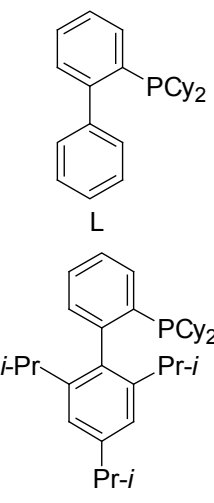

XPhos<smiles>Cc1ccccc1-c1ccccc1P(=O)(O)O</smiles>

MePhos<smiles>COc1ccc(OC)c(-c2c(P)cc(C(C)C)cc2P)c1P</smiles>

BrettPhos<smiles>COc1ccccc1-c1ccccc1OC</smiles>

SPhos

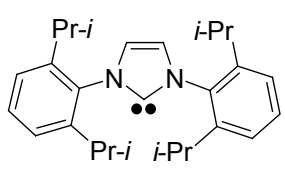

$\operatorname{IPr}$
Scheme 1
表 1 反应条件优化 ${ }^{a}$

Table 1 Reaction conditions optimization

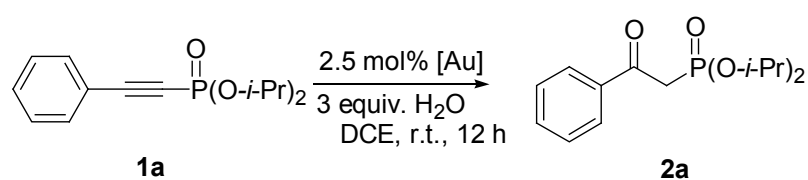

\begin{tabular}{|c|c|c|c|}
\hline Entry & Gold catalyst & Solvent & Yield $^{b, c} / \%$ \\
\hline 1 & $\mathrm{Ph}_{3} \mathrm{PAuNTf}_{2}$ & DCE & 73 \\
\hline 2 & $\left(4-\mathrm{MeOC}_{6} \mathrm{H}_{4}\right)_{3} \mathrm{PAuNTf}_{2}$ & DCE & 74 \\
\hline 3 & $\left(4-\mathrm{CF}_{3} \mathrm{C}_{6} \mathrm{H}_{4}\right)_{3} \mathrm{PAuNTf}_{2}$ & DCE & 73 \\
\hline 4 & IPrAuNTf & DCE & 70 \\
\hline 5 & $\mathrm{Et}_{3} \mathrm{PAuNTf}_{2}$ & DCE & 59 \\
\hline 5 & $\mathrm{Cy}_{3} \mathrm{PAuNTf}_{2}$ & DCE & 68 \\
\hline 6 & $\mathrm{LAuNTf}_{2}$ & DCE & 88 \\
\hline 7 & MePhosAuNTf $_{2}$ & DCE & 92 \\
\hline 8 & SPhosAuNTf $_{2}$ & DCE & 94 \\
\hline 9 & $\mathrm{XPhosAuNTf}_{2}$ & DCE & 98 \\
\hline 10 & BrettphosAuNTf ${ }_{2}$ & DCE & 98 \\
\hline 11 & XPhosAuCl & DCE & 80 \\
\hline 12 & $\mathrm{XPhosAuBF}_{4}$ & DCE & 90 \\
\hline $13^{e}$ & $\mathrm{XPhosAuSbF}_{6}$ & DCE & $98(92)$ \\
\hline 14 & $\mathrm{XPhosAuPF}_{6}$ & DCE & 95 \\
\hline 15 & XPhosAuOTf & DCE & 89 \\
\hline 16 & XPhosAuOTs & DCE & 87 \\
\hline $17^{d}$ & $\mathrm{XPhosAuSbF}_{6}$ & DCE & 83 \\
\hline 18 & $\mathrm{XPhosAuSbF}_{6}$ & $\mathrm{CH}_{3} \mathrm{OH}$ & 88 \\
\hline 19 & $\mathrm{XPhosAuSbF}_{6}$ & $\mathrm{CH}_{3} \mathrm{OH} / \mathrm{H}_{2} \mathrm{O}$ & 86 \\
\hline 20 & $\mathrm{XPhosAuSbF}_{6}$ & THF & 67 \\
\hline 21 & $\mathrm{XPhosAuSbF}_{6}$ & Acetone & 50 \\
\hline 22 & $\mathrm{XPhosAuSbF}_{6}$ & Toluene & - \\
\hline 23 & $\mathrm{XPhosAuSbF}_{6}$ & $\mathrm{CH}_{3} \mathrm{CN}$ & - \\
\hline 24 & $\mathrm{XPhosAuSbF}_{6}$ & DMF & - \\
\hline 25 & $\mathrm{XPhosAuSbF}_{6}$ & DMSO & - \\
\hline 26 & $\begin{array}{l}10 \mathrm{~mol}^{\%} \mathrm{XPhos}+ \\
10 \mathrm{~mol}^{\circ} \mathrm{AgSbF}_{6}\end{array}$ & DCE & - \\
\hline 27 & $\begin{array}{l}10 \mathrm{~mol} \% \mathrm{XPhos}+ \\
10 \mathrm{~mol} \% \mathrm{AgNO}_{3}\end{array}$ & DCE & - \\
\hline 28 & $\begin{array}{l}10 \mathrm{~mol} \% \text { XPhos }+ \\
10 \mathrm{~mol}^{\circ} \text { AgNTf }_{2}\end{array}$ & DCE & - \\
\hline 29 & $\begin{array}{l}10 \mathrm{~mol} \% \mathrm{XPhos}+ \\
10 \mathrm{~mol} \% \mathrm{Cu}(\mathrm{OTf})_{2}\end{array}$ & DCE & - \\
\hline 30 & $\begin{array}{l}10 \mathrm{~mol} \% \mathrm{XPhos}+ \\
10 \mathrm{~mol} \% \mathrm{Cu}\left(\mathrm{NO}_{3}\right)_{2}\end{array}$ & DCE & 一 \\
\hline 31 & $\mathrm{HNTf}_{2}$ & DCE & - \\
\hline 32 & $\mathrm{MsOH}$ & DCE & - \\
\hline 33 & TFA & DCE & 一 \\
\hline
\end{tabular}

${ }^{a}$ Alkynylphosphonate (1a) $(1 \mathrm{mmol}), \mathrm{H}_{2} \mathrm{O}(3 \mathrm{mmol})$, gold catalyst $(2.5 \mathrm{~mol} \%)$, DCE $(1 \mathrm{~mL})$ in the open flask at room temperature. ${ }^{b}$ The reaction progress was monitored by thin layer chromatography. ${ }^{c}$ Estimated by ${ }^{1} \mathrm{H}$ NMR spectroscopy using 1,3,5-trimethoxybenzene as an internal reference and the number in parentheses is isolated yield. ${ }^{d} 1.5 \mathrm{~mol} \% \mathrm{XPhosAuSbF}_{6}$ was used and the reaction was run for $40 \mathrm{~h}$

使用 BrettPhosAuNTf 2 作为催化剂的水合反应底物核磁 转化率与使用 $\mathrm{XPhosAuNTf}_{2}$ 时反应的核磁理论转化率 
接近. 其次我们考察了金催化剂共轭阴离子对反应转化 率的影响, 在所测试的 5 种阴离子中(Entries 11 16), 含有 $\mathrm{SbF}_{6}^{-}$和 $\mathrm{NTf}_{2}^{-}$的金催化剂表现出相同的催化性能. 考虑到金催化剂的价格因素, 最终我们选择 $\mathrm{XPhosAuSbF}_{6}$ 作为该反应催化剂. 本文进一步研究了减 少催化剂使用量对反应的影响, 研究发现 $1.5 \mathrm{~mol} \%$ 的金 催化剂也可以催化反应进行. 延长反应时间至 $40 \mathrm{~h}$, 此 时约有 $12 \%$ 的炔基膦酸酯未反应, 相应产物的核磁收率 为 $83 \%$ (Entry 17), 继续延长反应时间至 $72 \mathrm{~h}$, 产率没有 提高.

之后本文考察了溶剂效应对该反应的影响. 如表 1 所示, 溶剂效应在反应中发挥着重要做作用. 使用水合 反应，常用的甲醇、甲醇/水 $(V: V=10: 1)$ 、四氢呋喃 和丙酮时, 反应的转化率均有所降低(Entries 18 21), 而使用甲苯、乙腈、 $N, N$-二甲基甲酰胺及二甲基亚砜时 无法得到相应的水合产物, 原料几乎没有减少(Entries 22 25). 研究还发现银盐、铜盐及质子酸都不能催化炔 基膦酸酯的水合反应(Entries 26 33).

通过上述的条件优化, 我们最终选择在室温下, 向 1,2-二氯乙烷溶剂中加入 $2.5 \mathrm{~mol} \% \mathrm{XPhosAuSbF}_{6}$ 催化炔 基膦酸酯的水合反应(Talbe 1, Entry 13).

\section{2 反应适用性研究}

在最优化的反应条件下，对多种炔基膦酸酯的水合 反应进行了研究. 阳离子金催化炔基膦酸酯水合反应不 需加入任何酸碱，室温下进行，反应条件非常温和，所 以本反应也应用于对酸、碱及高温敏感的底物. 如表 2 所示, 芳炔基膦酸酯底物芳环的对位、间位存在吸电子 基团(氟、氯、溴和乙酰氧基)或供电子基团(甲基和甲氧 基)时, 底物都能近乎完全转化成相应的 $\beta$-羰基膦酸酯 化合物(Table 2, Entries 1 8). 在芳炔底物的邻位有甲 基或溴原子时，反应也能非常顺利地进行(Entries 9, 10). 此外使用具有生物活性的杂环底物, 如噻吩、吡啶、胡 椒基、荎都能高产率地得到相应的水合产物(Entries $11 \sim 14)$. 酯肪烃底物的研究结果表明, 该反应能很好 地兼容含多种重要官能团的氯代炔烃，包括烷基(Entry 15)、苯乙基(Entry 16)、卤素(Entry 17)、腈基(Entry 18)、 环已烯基(Entry 19)和环已基(Entry 20). 研究还发现其 他的膦酸酯底物，如二甲氧基、二乙氧基、二正丁氧基 和芐氧基(Entires 21 24)都可以发生水合反应，高产率 地得到相应的 $\beta$-羰基膦酸酯目标化合物.

表 2 反应范围 ${ }^{a}$

Table 2 The reaction scope

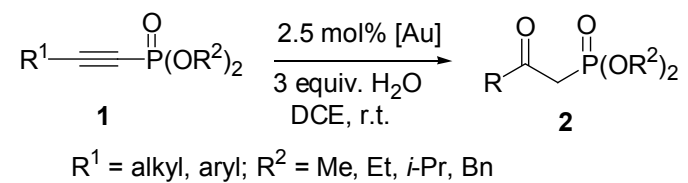

Entry




\begin{tabular}{|c|c|c|c|c|c|}
\hline Entry & Product & Yield ${ }^{b} / \%$ & Entry & Product & Yield $^{b} / \%$ \\
\hline 9 & $2 j$ & 94 & 10 & $2 k$ & 92 \\
\hline 11 & 2 I & 96 & 12 & $2 m$ & 98 \\
\hline 13 & $2 n$ & 92 & 14 & 20 & 96 \\
\hline 15 & $2 p$ & 95 & 16 & $2 q$ & 94 \\
\hline 17 & $2 r$ & 94 & 18 & $2 s$ & 97 \\
\hline 19 & $2 t$ & 97 & 20 & $2 u$ & 97 \\
\hline 21 & $2 v$ & 94 & 22 & $2 w$ & 95 \\
\hline 23 & $2 x$ & 96 & 24 & $2 y$ & 95 \\
\hline
\end{tabular}

${ }^{a}$ Alkynylphosphonate (1) (3 mmol), $\mathrm{H}_{2} \mathrm{O}(9 \mathrm{mmol}), \mathrm{XPhosAuSbF}_{6}(2.5 \mathrm{~mol} \%)$, DCE $3 \mathrm{~mL}$ in the open flask at room temperature. ${ }^{b}$ Isolated yield.

\section{3 金催化炔基膦酸酯水合反应的应用}

二氢嘧啶酮膦酸酯化合物是一类重要的含膦杂环 化合物, 具有广泛的生物活性 ${ }^{[46]}$. 本文以炔基膦酸酯为 初始原料, 通过金催化水合反应得到化合物 $\mathbf{2 v}$, 将所得 反应液过滤旋干后直接用于 Biginelli 多组反应 ${ }^{[47]}$ 即得 到二氢嘧啶酮膦酸酯化合物, 总产率 $84 \%$ (Scheme 2).

\section{4 金催化炔基膦酸酯水合反应的可能机理}

基于文献报道 ${ }^{[2]}$ 和本文实验结果，推测金催化炔基 膦酸酯水合反应可能的反应机理见 Scheme 3. 首先, 阳 离子金催化剂活化炔基膦酸酯生成中间体 $\mathbf{A}$. 金的拉电 子作用使炔基偏正电性，水分子与中间体 $\mathbf{A}$ 发生亲核加

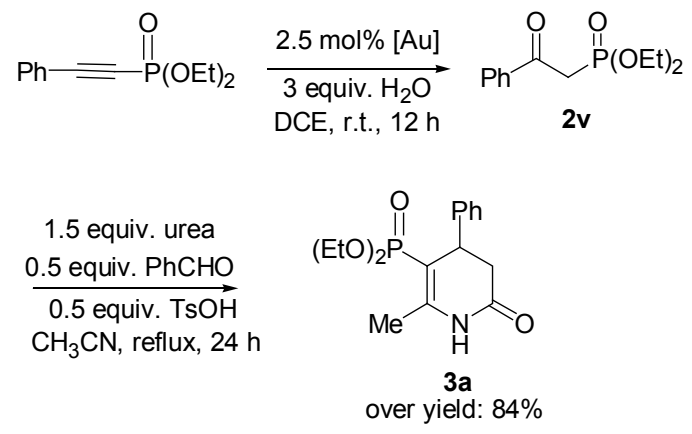

Scheme 2

成反应生成中间体 $\mathbf{B}$; 中间体 $\mathbf{B}$ 不稳定，易发生酮一烯醇 互变异构生成更稳定的酮式结构，最后质子去金化得到 
$\beta$-羰基膦酸酯化合物.

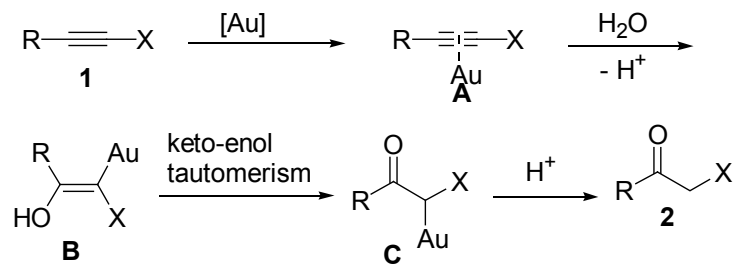

Scheme 3

\section{2 结论}

我们研究了阳离子金催化剂催化炔基膦酸酯化合 物的水合反应, 利用 XPhosAuSbF 金配合物开发了一种 操作简便、原子经济性高的合成 $\beta$-羊炭基膦酸酯化合物的 新方法. 该方法无需加入酸性或碱性助催化剂、无需惰 性气体保护，不使用、不产生有毒危害试剂，反应条件 温和且具有广泛的反应底物普适性. 该研究工作不仅丰 富了金催化炔烃水合反应体系, 并为含有 $\beta$-羰基膦酸酯 结构的天然产物及药物分子合成提供了新的途径.

\section{3 实验部分}

\section{1 试剂与仪器}

金催化剂均购自百灵威, 反应底物均购自阿拉丁、 百灵威、安耐吉等公司, 所有药品和试剂均为分析纯. ${ }^{1} \mathrm{H}$ $\mathrm{NMR}$ 和 ${ }^{13} \mathrm{C} \mathrm{NMR}$ (内标为 TMS, 溶剂为 $\mathrm{CDCl}_{3}$ ) 使用 Varian INOVA-400 型核磁共振仪测定. MS 使用 MAT95XP 型高分辨质谱仪测定.

\section{2 目标产物的合成}

室温条件下，在 $10 \mathrm{~mL}$ 圆底烧瓶中依次加入 0.3

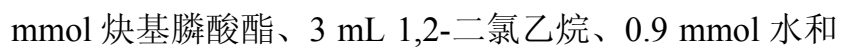
$2.5 \mathrm{~mol}^{\circ} \mathrm{XPhosAuSbF}_{6}(0.0075 \mathrm{mmol}, 6.8 \mathrm{mg})$, 室温摚 拌反应一段时间后 TLC 监控反应完成. 减压旋去溶剂, 得到粘稠液或固体, 混合物再经柱层析(乙酸乙酯/石油 醚 $2: 1 \sim 1: 1)$ 分离提纯得目标产物 $\mathbf{2 a} \sim 2 \mathbf{y}$.

(2-苯乙基-2-氧代)膦酸二异丙酯 $(\mathbf{2 a})^{[40]}$ : 淡黄色油 状物. ${ }^{1} \mathrm{H}$ NMR $\left(400 \mathrm{MHz}, \mathrm{CDCl}_{3}\right) \delta: 8.00(\mathrm{~d}, J=7.7 \mathrm{~Hz}$, $2 \mathrm{H}), 7.58(\mathrm{t}, J=7.3 \mathrm{~Hz}, 1 \mathrm{H}), 7.48(\mathrm{t}, J=7.7 \mathrm{~Hz}, 2 \mathrm{H})$, $4.74 \sim 4.69(\mathrm{~m}, 2 \mathrm{H}), 3.58(\mathrm{~d}, J=23.1 \mathrm{~Hz}, 2 \mathrm{H}), 1.27$ (t, $J=$ $5.1 \mathrm{~Hz}, 12 \mathrm{H}) ;{ }^{13} \mathrm{C}$ NMR $\left(100 \mathrm{MHz}, \mathrm{CDCl}_{3}\right) \delta: 192.1$, $136.8,133.4,129.2,128.5,71.6\left(\mathrm{~d}, J_{\mathrm{C}-\mathrm{P}}=6.8 \mathrm{~Hz}\right), 39.8(\mathrm{~d}$, $\left.J_{\mathrm{C}-\mathrm{P}}=130.0 \mathrm{~Hz}\right), 23.8,23.6$; HRMS calcd for $\mathrm{C}_{14} \mathrm{H}_{21} \mathrm{O}_{4} \mathrm{NaP}$ $(\mathrm{M}+\mathrm{Na})^{+}$307.1075, found 307.1068.

[2-(4'-甲基苯乙基)-2-氧代]膦酸二异丙酯 $(2 \mathbf{b})^{[41]}$ : 淡黄色油状物. ${ }^{1} \mathrm{H}$ NMR (400 MHz, $\left.\mathrm{CDCl}_{3}\right) \delta: 7.93$ (d, $J=8.0 \mathrm{~Hz}, 2 \mathrm{H}), 7.25(\mathrm{~d}, J=8.0 \mathrm{~Hz}, 2 \mathrm{H}), 4.74 \sim 4.68(\mathrm{~m}$, $2 \mathrm{H}), 3.57$ (d, $J=22.7 \mathrm{~Hz}, 2 \mathrm{H}), 2.42$ (s, 3H), 1.27 (t, $J=5.9$
$\mathrm{Hz}, 12 \mathrm{H}) ;{ }^{13} \mathrm{C}$ NMR $\left(100 \mathrm{MHz}, \mathrm{CDCl}_{3}\right) \delta: 191.5,144.5$, $134.4,129.2,129.3,71.5\left(\mathrm{~d}, J_{\mathrm{C}-\mathrm{P}}=6.8 \mathrm{~Hz}\right), 39.8\left(\mathrm{~d}, J_{\mathrm{C}-\mathrm{P}}=\right.$ $128.9 \mathrm{~Hz}), 23.8\left(\mathrm{~d}, J_{\mathrm{C}-\mathrm{P}}=3.4 \mathrm{~Hz}\right), 23.6\left(\mathrm{~d}, J_{\mathrm{C}-\mathrm{P}}=5.7 \mathrm{~Hz}\right)$, 21.8; HRMS calcd for $\mathrm{C}_{15} \mathrm{H}_{23} \mathrm{O}_{4} \mathrm{NaP}(\mathrm{M}+\mathrm{Na})^{+} 321.1232$, found 321.1242 .

[2-(4'-甲氧基苯乙基)-2-氧代]膦酸二异丙酯 $(2 \mathbf{c})^{[40]}$ : 淡黄色油状物. ${ }^{1} \mathrm{H}$ NMR $\left(400 \mathrm{MHz}, \mathrm{CDCl}_{3}\right) \delta: 7.97$ (d, $J=8.8 \mathrm{~Hz}, 2 \mathrm{H}), 6.88(\mathrm{~d}, J=8.8 \mathrm{~Hz}, 2 \mathrm{H}), 4.71 \sim 4.66(\mathrm{~m}$, $2 \mathrm{H}), 3.82$ (s, $3 \mathrm{H}), 3.48$ (d, $J=23.2 \mathrm{~Hz}, 2 \mathrm{H}), 1.25$ (t, $J=6.1$ $\mathrm{Hz}, 12 \mathrm{H}) ;{ }^{13} \mathrm{C}$ NMR $\left(100 \mathrm{MHz}, \mathrm{CDCl}_{3}\right) \delta: 190.4\left(\mathrm{~d}, J_{\mathrm{C}-\mathrm{P}}=\right.$ $6.9 \mathrm{~Hz}), 163.7,131.6,129.7,113.5,71.4\left(\mathrm{~d}, J_{\mathrm{C}-\mathrm{P}}=6.8 \mathrm{~Hz}\right)$, $55.5,39.7\left(\mathrm{~d}, J_{\mathrm{C}-\mathrm{P}}=130.0 \mathrm{~Hz}\right), 23.8\left(\mathrm{~d}, J_{\mathrm{C}-\mathrm{P}}=3.4 \mathrm{~Hz}\right), 23.6$ $\left(\mathrm{d}, J_{\mathrm{C}-\mathrm{P}}=4.6 \mathrm{~Hz}\right)$; HRMS calcd for $\mathrm{C}_{15} \mathrm{H}_{24} \mathrm{O}_{5} \mathrm{P}(\mathrm{M}+\mathrm{H})^{+}$ 315.1361 , found 315.1365 .

[2-(3'-甲基苯乙基)-2-氧代]膦酸二异丙酯 $(\mathbf{2 d})^{[40] \text { : }}$ 淡黄色油状物. ${ }^{1} \mathrm{H}$ NMR $\left(400 \mathrm{MHz}, \mathrm{CDCl}_{3}\right) \delta: 7.83$ (d, $J=8.0 \mathrm{~Hz}, 2 \mathrm{H}), 7.38(\mathrm{~d}, J=7.7 \mathrm{~Hz}, 1 \mathrm{H}), 7.37$ (t, $J=7.5$ $\mathrm{Hz}, 1 \mathrm{H}), 4.75 \sim 4.70(\mathrm{~m}, 2 \mathrm{H}), 3.59(\mathrm{~d}, J=22.7 \mathrm{~Hz}, 2 \mathrm{H})$, 2.43 (s, 3H), 1.28 (d, $J=2.9 \mathrm{~Hz}, 6 \mathrm{H}$, overlap), 1.28 (d, $J=$ $2.9 \mathrm{~Hz}, 6 \mathrm{H}$, overlap); ${ }^{13} \mathrm{C}$ NMR $\left(100 \mathrm{MHz}, \mathrm{CDCl}_{3}\right) \delta$ : $192.3\left(\mathrm{~d}, J_{\mathrm{C}-\mathrm{P}}=6.8 \mathrm{~Hz}\right), 138.3,136.8,134.1,129.7,128.5$, $126.4,71.3\left(\mathrm{~d}, J_{\mathrm{C}-\mathrm{P}}=6.8 \mathrm{~Hz}\right), 39.8\left(\mathrm{~d}, J_{\mathrm{C}-\mathrm{P}}=130.0 \mathrm{~Hz}\right)$, $23.8\left(\mathrm{~d}, J_{\mathrm{C}-\mathrm{P}}=3.4 \mathrm{~Hz}\right), 23.8\left(\mathrm{~d}, J_{\mathrm{C}-\mathrm{P}}=4.6 \mathrm{~Hz}\right), 21.3$; HRMS calcd for $\mathrm{C}_{15} \mathrm{H}_{23} \mathrm{O}_{4} \mathrm{NaP}(\mathrm{M}+\mathrm{Na})^{+}$321.1232, found 321.1231 .

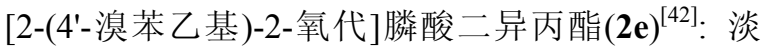
黄色油状物. ${ }^{1} \mathrm{H}$ NMR $\left(400 \mathrm{MHz}, \mathrm{CDCl}_{3}\right) \delta: 7.88(\mathrm{~d}, J=$ $8.5 \mathrm{~Hz}, 2 \mathrm{H}$ ), 7.62 (br.t, $J=8.4 \mathrm{~Hz}, 2 \mathrm{H}), 4.76 \sim 4.70(\mathrm{~m}$, $2 \mathrm{H}), 3.54$ (d, $J=22.7 \mathrm{~Hz}, 2 \mathrm{H}), 1.28$ (d, $J=3.3 \mathrm{~Hz}, 6 \mathrm{H}$, overlap), 1.29 (d, $J=3.3 \mathrm{~Hz}, 6 \mathrm{H}$, overlap); ${ }^{13} \mathrm{C}$ NMR (100 $\left.\mathrm{MHz}, \mathrm{CDCl}_{3}\right) \delta: 191.2$ (d, $\left.J_{\mathrm{C}-\mathrm{P}}=6.8 \mathrm{~Hz}\right), 135.3,131.9$, $130.7,128.9,71.5\left(\mathrm{~d}, J_{\mathrm{C}-\mathrm{P}}=6.8 \mathrm{~Hz}\right), 39.8\left(\mathrm{~d}, J_{\mathrm{C}-\mathrm{P}}=128.9\right.$ $\mathrm{Hz}), 23.8\left(\mathrm{~d}, J_{\mathrm{C}-\mathrm{P}}=3.4 \mathrm{~Hz}\right), 23.7\left(\mathrm{~d}, J_{\mathrm{C}-\mathrm{P}}=4.6 \mathrm{~Hz}\right)$; HRMS calcd for $\mathrm{C}_{14} \mathrm{H}_{20} \mathrm{O}_{4} \mathrm{NaPBr}(\mathrm{M}+\mathrm{Na})^{+} 385.0180$, found 385.0188 .

[2-(4'-氯苯乙基)-2-氧代]膦酸二异丙酯 $(2 \mathbf{f})^{[41]}$ : 淡黄 色油状物. ${ }^{1} \mathrm{H}$ NMR $\left(400 \mathrm{MHz}, \mathrm{CDCl}_{3}\right) \delta: 7.97 \sim 7.95(\mathrm{~m}$, $2 \mathrm{H}), 7.46 \sim 7.44(\mathrm{~m}, 2 \mathrm{H}), 4.74 \sim 4.68(\mathrm{~m}, 2 \mathrm{H}), 3.56(\mathrm{~d}, J=$ $22.7 \mathrm{~Hz}, 2 \mathrm{H}), 1.28$ (d, $J=3.3 \mathrm{~Hz}, 6 \mathrm{H}$, overlap), 1.29 (d, $J=3.3 \mathrm{~Hz}, 6 \mathrm{H}$, overlap); ${ }^{13} \mathrm{C}$ NMR $\left(100 \mathrm{MHz}, \mathrm{CDCl}_{3}\right) \delta$ : $190.8\left(\mathrm{~d}, \quad J_{\mathrm{C}-\mathrm{P}}=5.7 \mathrm{~Hz}\right), 140.1,135.1,130.5,128.7,71.7$ $\left(\mathrm{d}, J_{\mathrm{C}-\mathrm{P}}=6.8 \mathrm{~Hz}\right), 39.8\left(\mathrm{~d}, J_{\mathrm{C}-\mathrm{P}}=128.9 \mathrm{~Hz}\right), 23.8\left(\mathrm{~d}, J_{\mathrm{C}-\mathrm{P}}=\right.$ $3.4 \mathrm{~Hz}), 23.8\left(\mathrm{~d}, J_{\mathrm{C}-\mathrm{P}}=5.7 \mathrm{~Hz}\right)$; HRMS calcd for $\mathrm{C}_{14} \mathrm{H}_{21^{-}}$ $\mathrm{O}_{4} \mathrm{PCl}(\mathrm{M}+\mathrm{H})^{+}$319.0866, found 319.0866.

[2-(4'-氟苯乙基)-2-氧代]膦酸二异丙酯 $(2 \mathrm{~g})^{[40]}$ : 淡 
黄色油状物. ${ }^{1} \mathrm{H}$ NMR (400 MHz, $\left.\mathrm{CDCl}_{3}\right) \delta: 8.08 \sim 8.06$ $(\mathrm{m}, 2 \mathrm{H}), 7.18 \sim 7.14(\mathrm{~m}, 2 \mathrm{H}), 4.75 \sim 4.70(\mathrm{~m}, 2 \mathrm{H}), 3.58(\mathrm{~d}$, $J=23.1 \mathrm{~Hz}, 2 \mathrm{H}), 1.31$ (d, $J=3.7 \mathrm{~Hz}, 6 \mathrm{H}$, overlap), 1.28 (d, $J=3.7 \mathrm{~Hz}, 6 \mathrm{H}$, overlap); ${ }^{13} \mathrm{C} \mathrm{NMR}\left(100 \mathrm{MHz}, \mathrm{CDCl}_{3}\right) \delta$ : $190.4\left(\mathrm{~d}, J_{\mathrm{C}-\mathrm{P}}=6.8 \mathrm{~Hz}\right), 166.8,165.2,133.1,132.1,131.8$, $115.7,115.6,71.5\left(\mathrm{~d}, J_{\mathrm{C}-\mathrm{P}}=6.8 \mathrm{~Hz}\right), 39.8\left(\mathrm{~d}, J_{\mathrm{C}-\mathrm{P}}=128.9\right.$ $\mathrm{Hz}), 23.8\left(\mathrm{~d}, J_{\mathrm{C}-\mathrm{P}}=3.4 \mathrm{~Hz}\right), 23.8\left(\mathrm{~d}, J_{\mathrm{C}-\mathrm{P}}=4.6 \mathrm{~Hz}\right)$; HRMS calcd for $\mathrm{C}_{14} \mathrm{H}_{20} \mathrm{O}_{4} \mathrm{NaPF}(\mathrm{M}+\mathrm{Na})^{+}$325.0981, found 325.0978 .

[2-(4'-乙酰氧基苯乙基)-2-氧代]膦酸二异丙酯 $(\mathbf{2 h})^{[40]}$ : 淡黄色油状物. ${ }^{1} \mathrm{H}$ NMR $\left(400 \mathrm{MHz} \mathrm{CDCl}_{3}\right) \delta$ : $8.05(\mathrm{~d}, J=8.5 \mathrm{~Hz}, 2 \mathrm{H}), 7.22$ (d, $J=8.4 \mathrm{~Hz}, 2 \mathrm{H}), 4.74 \sim$ $4.69(\mathrm{~m}, 2 \mathrm{H}), 3.58(\mathrm{~d}, J=22.7 \mathrm{~Hz}, 2 \mathrm{H}), 2.33(\mathrm{~s}, 3 \mathrm{H})$, 1.28 (d, $J=2.9 \mathrm{~Hz}, 6 \mathrm{H}$, overlap), 1.27 (d, $J=2.6 \mathrm{~Hz}, 6 \mathrm{H}$, overlap); ${ }^{13} \mathrm{C}$ NMR (100 MHz, $\left.\mathrm{CDCl}_{3}\right) \delta: 190.7$ (d, $J_{\mathrm{C}-\mathrm{P}}=$ $6.8 \mathrm{~Hz}), 168.8,154.8,134.2,130.7,121.8,71.7\left(\mathrm{~d}, J_{\mathrm{C}-\mathrm{P}}=\right.$ $6.8 \mathrm{~Hz}), 39.8\left(\mathrm{~d}, J_{\mathrm{C}-\mathrm{P}}=128.9 \mathrm{~Hz}\right), 23.8\left(\mathrm{~d}, J_{\mathrm{C}-\mathrm{P}}=4.6 \mathrm{~Hz}\right)$, $23.6\left(\mathrm{~d}, J_{\mathrm{C}-\mathrm{P}}=4.6 \mathrm{~Hz}\right), 21.2$; HRMS calcd for $\mathrm{C}_{16} \mathrm{H}_{23}-$ $\mathrm{O}_{6} \mathrm{NaP}(\mathrm{M}+\mathrm{Na})^{+}$365.1130, found 365.1133.

[2-(3'-溴苯乙基)-2-氧代]膦酸二乙酯 $(\mathbf{2 i})^{[42]}$ : 淡黄色 油状物. ${ }^{1} \mathrm{H} \mathrm{NMR}\left(400 \mathrm{MHz}, \mathrm{CDCl}_{3}\right) \delta: 8.12(\mathrm{t}, J=2.0 \mathrm{~Hz}$, $1 \mathrm{H}), 7.93(\mathrm{dt}, J=8.0 \mathrm{~Hz}, 1.2 \mathrm{~Hz}, 1 \mathrm{H}), 7.70 \sim 7.67(\mathrm{~m}, 1 \mathrm{H})$, $7.32(\mathrm{t}, J=8.0 \mathrm{~Hz}, 1 \mathrm{H}), 4.13 \sim 4.05(\mathrm{~m}, 4 \mathrm{H}), 3.58\left(\mathrm{~d}, J_{\mathrm{H}-\mathrm{P}}\right.$ $=22.4 \mathrm{~Hz}, 2 \mathrm{H}), 1.26(\mathrm{t}, J=7.2 \mathrm{~Hz}, 6 \mathrm{H}) ;{ }^{13} \mathrm{C} \mathrm{NMR}(100$ $\left.\mathrm{MHz}, \mathrm{CDCl}_{3}\right) \delta: 190.55\left(\mathrm{~d}, J_{\mathrm{C}-\mathrm{P}}=6.5 \mathrm{~Hz}\right), 138.09,136.36$, $131.88,130.08,127.56,122.81,62.66\left(\mathrm{~d}, J_{\mathrm{C}-\mathrm{P}}=6.5 \mathrm{~Hz}\right)$, $38.56\left(\mathrm{~d}, J_{\mathrm{C}-\mathrm{P}}=128.3 \mathrm{~Hz}\right), 16.13\left(\mathrm{~d}, J_{\mathrm{C}-\mathrm{P}}=5.8 \mathrm{~Hz}\right)$; HRMS calcd for $\mathrm{C}_{12} \mathrm{H}_{16} \mathrm{BrO}_{4} \mathrm{P}(\mathrm{M}+\mathrm{H})^{+}$333.9968, found 333.9964.

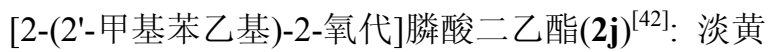
色油状物. ${ }^{1} \mathrm{H}$ NMR (400 MHz, $\left.\mathrm{CDCl}_{3}\right) \delta: 7.72$ (d, $J=7.7$ $\mathrm{Hz}, 1 \mathrm{H}), 7.38$ (t, $J=7.5 \mathrm{~Hz}, 1 \mathrm{H}), 7.27 \sim 7.22(\mathrm{~m}, 2 \mathrm{H})$, $4.74 \sim 7.69(\mathrm{~m}, 2 \mathrm{H}), 3.55(\mathrm{~d}, J=22.7 \mathrm{~Hz}, 2 \mathrm{H}), 2.52$ (s, $3 \mathrm{H}), 1.27$ (d, $J=6.2 \mathrm{~Hz}, 6 \mathrm{H}$, overlap), 1.25 (d, $J=6.2 \mathrm{~Hz}$, $6 \mathrm{H}$, overlap); ${ }^{13} \mathrm{C}$ NMR (100 MHz, $\left.\mathrm{CDCl}_{3}\right) \delta$ : $195.2(\mathrm{~d}$, $\left.J_{\mathrm{C}-\mathrm{P}}=6.9 \mathrm{~Hz}\right), 138.8,137.8,131.9$, 131.5, 129.5, 125.6, $71.2\left(\mathrm{~d}, J_{\mathrm{C}-\mathrm{P}}=6.8 \mathrm{~Hz}\right), 42.4\left(\mathrm{~d}, J_{\mathrm{C}-\mathrm{P}}=128.9 \mathrm{~Hz}\right), 23.8(\mathrm{~d}$, $\left.J_{\mathrm{C}-\mathrm{P}}=3.4 \mathrm{~Hz}\right), 23.6\left(\mathrm{~d}, J_{\mathrm{C}-\mathrm{P}}=5.7 \mathrm{~Hz}\right), 21.2$; HRMS calcd for $\mathrm{C}_{15} \mathrm{H}_{23} \mathrm{O}_{4} \mathrm{NaP}(\mathrm{M}+\mathrm{Na})^{+}$321.1232, found 321.1230.

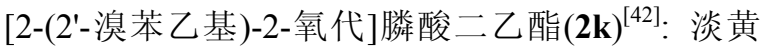
色油状物. ${ }^{1} \mathrm{H}$ NMR (400 MHz, $\left.\mathrm{CDCl}_{3}\right) \delta: 8.11(\mathrm{t}, J=2.0$ $\mathrm{Hz}, 1 \mathrm{H}), 7.93(\mathrm{dt}, J=8.0 \mathrm{~Hz}, 1.2 \mathrm{~Hz}, 1 \mathrm{H}), 7.70 \sim 7.67(\mathrm{~m}$, $1 \mathrm{H}), 7.33$ (t, $J=8.0 \mathrm{~Hz}, 1 \mathrm{H}), 4.14 \sim 4.06(\mathrm{~m}, 4 \mathrm{H}), 3.57$ (d, $\left.J_{\mathrm{H}-\mathrm{P}}=22.4 \mathrm{~Hz}, 2 \mathrm{H}\right), 1.26(\mathrm{t}, J=7.2 \mathrm{~Hz}, 6 \mathrm{H}) ;{ }^{13} \mathrm{C} \mathrm{NMR}$ $\left(100 \mathrm{MHz}, \mathrm{CDCl}_{3}\right) \delta: 190.55\left(\mathrm{~d}, J_{\mathrm{C}-\mathrm{P}}=6.5 \mathrm{~Hz}\right), 138.09$, $136.36,131.88,130.08,127.56,121.81,62.66\left(\mathrm{~d}, J_{\mathrm{C}-\mathrm{P}}=6.5\right.$ $\mathrm{Hz}), 38.56\left(\mathrm{~d}, J_{\mathrm{C}-\mathrm{P}}=128.3 \mathrm{~Hz}\right), 16.13\left(\mathrm{~d}, J_{\mathrm{C}-\mathrm{P}}=5.8 \mathrm{~Hz}\right.$ ); HRMS calcd for $\mathrm{C}_{12} \mathrm{H}_{16} \mathrm{BrO}_{4} \mathrm{P}(\mathrm{M}+\mathrm{H})^{+} 333.9965$, found 333.9964.

[2-(3'-乙基噻吩基)-2-氧代]膦酸二乙酯 $(21)^{[40]}$ : 淡黄 色油状物. ${ }^{1} \mathrm{H}$ NMR (400 MHz, $\left.\mathrm{CDCl}_{3}\right) \delta: 8.14(\mathrm{~d}, J=2.0$ $\mathrm{Hz}, 1 \mathrm{H}), 7.52(\mathrm{~d}, J=6.1 \mathrm{~Hz}, 1 \mathrm{H}), 7.25 \sim 7.24(\mathrm{~m}, 1 \mathrm{H})$, $4.11 \sim 4.04(\mathrm{~m}, 4 \mathrm{H}), 3.45\left(\mathrm{~d}, J_{\mathrm{H}-\mathrm{P}}=22.8 \mathrm{~Hz}, 2 \mathrm{H}\right), 1.23(\mathrm{t}$, $J=7.2 \mathrm{~Hz}, 6 \mathrm{H}) ;{ }^{13} \mathrm{C} \mathrm{NMR}\left(100 \mathrm{MHz}, \mathrm{CDCl}_{3}\right) \delta: 185.49$ (d, $\left.J_{\mathrm{C}-\mathrm{P}}=6.6 \mathrm{~Hz}\right), 141.78,134.26,127.18,126.30,62.58(\mathrm{~d}$, $\left.J_{\mathrm{C}-\mathrm{P}}=6.5 \mathrm{~Hz}\right), 39.89\left(\mathrm{~d}, J_{\mathrm{C}-\mathrm{P}}=128.4 \mathrm{~Hz}\right), 16.12\left(\mathrm{~d}, J_{\mathrm{C}-\mathrm{P}}=\right.$ $6.6 \mathrm{~Hz})$; HRMS calcd for $\mathrm{C}_{10} \mathrm{H}_{15} \mathrm{O}_{4} \mathrm{PS}(\mathrm{M}+\mathrm{H})^{+}, 262.0426$, found 262.0422 .

[2-(2'-乙基吡啶基)-2-氧代]膦酸二异丙酯 $(\mathbf{2 m})^{[40]}$ : 淡黄色油状物. ${ }^{1} \mathrm{H}$ NMR $\left(400 \mathrm{MHz}, \mathrm{CDCl}_{3}\right) \delta: 8.71(\mathrm{~d}$, $J=4.4 \mathrm{~Hz}, 1 \mathrm{H}), 8.05$ (d, $J=7.7 \mathrm{~Hz}, 1 \mathrm{H}), 7.82$ (t, $J=7.7$ $\mathrm{Hz}, 1 \mathrm{H}), 7.49 \sim 7.47(\mathrm{~m}, 1 \mathrm{H}), 4.76 \sim 4.70(\mathrm{~m}, 2 \mathrm{H}), 3.99(\mathrm{~d}$, $J=22.7 \mathrm{~Hz}, 2 \mathrm{H}), 1.29$ (d, $J=5.8 \mathrm{~Hz}, 6 \mathrm{H}$, overlap), 1.25 (d, $J=6.2 \mathrm{~Hz}, 6 \mathrm{H}$, overlap); ${ }^{13} \mathrm{C} \mathrm{NMR}\left(100 \mathrm{MHz}, \mathrm{CDCl}_{3}\right) \delta$ : $194.1,153.1,148.8,136.9,127.2,122.3,71.1\left(\mathrm{~d}, J_{\mathrm{C}-\mathrm{P}}=5.7\right.$ $\mathrm{Hz}), 37.1\left(\mathrm{~d}, J_{\mathrm{C}-\mathrm{P}}=130.0 \mathrm{~Hz}\right), 24.2\left(\mathrm{~d}, J_{\mathrm{C}-\mathrm{P}}=3.4 \mathrm{~Hz}\right), 23.6$ $\left(\mathrm{d}, J_{\mathrm{C}-\mathrm{P}}=5.7 \mathrm{~Hz}\right)$; HRMS calcd for $\mathrm{C}_{13} \mathrm{H}_{21} \mathrm{NO}_{4} \mathrm{P}(\mathrm{M}+\mathrm{H})^{+}$ 286.1208, found 286.1219.

[2-(3',4'-亚甲二氧基苯乙基)-2-氧代]膦酸二乙酯 $(2 n)^{[41]}$ : 淡黄色油状物. ${ }^{1} \mathrm{H}$ NMR $\left(400 \mathrm{MHz}, \mathrm{CDCl}_{3}\right) \delta$ : 7.58 (d, $J=8.4 \mathrm{~Hz}, 1 \mathrm{H}), 7.42$ (s, 1H), 6.83 (d, $J=8.4 \mathrm{~Hz}$, $1 \mathrm{H}), 6.02(\mathrm{~s}, 2 \mathrm{H}), 4.15 \sim 4.07(\mathrm{~m}, 4 \mathrm{H}), 3.53\left(\mathrm{~d}, J_{\mathrm{H}-\mathrm{P}}=22.8\right.$ $\mathrm{Hz}, 2 \mathrm{H}), 1.24$ (t, $J=7.2 \mathrm{~Hz}, 6 \mathrm{H}) ;{ }^{13} \mathrm{C} \mathrm{NMR}(100 \mathrm{MHz}$, $\left.\mathrm{CDCl}_{3}\right) \delta: 189.72\left(\mathrm{~d}, J_{\mathrm{C}-\mathrm{P}}=6.6 \mathrm{~Hz}\right), 152.26,148.18$, $131.32,125.97,108.36,107.75,101.93,62.58\left(\mathrm{~d}, J_{\mathrm{C}-\mathrm{P}}=6.6\right.$ $\mathrm{Hz}), 38.24\left(\mathrm{~d}, J_{\mathrm{C}-\mathrm{P}}=129.8 \mathrm{~Hz}\right), 16.16\left(\mathrm{~d}, J_{\mathrm{C}-\mathrm{P}}=6.6 \mathrm{~Hz}\right) ;{ }^{31} \mathrm{P}$ NMR (162 MHz, $\left.\mathrm{CDCl}_{3}\right) \delta$ : 20.22; HRMS calcd for $\mathrm{C}_{13} \mathrm{H}_{17} \mathrm{O}_{6} \mathrm{P}(\mathrm{M}+\mathrm{H})^{+} \quad 300.0765$, found 300.0759 .

(2-荟乙基-2-氧代)膦酸二异丙酯 $(\mathbf{2 0})^{[41]}$ : 淡黄色油 状物. ${ }^{1} \mathrm{H}$ NMR $\left(400 \mathrm{MHz}, \mathrm{CDCl}_{3}\right) \delta: 8.56(\mathrm{~s}, 1 \mathrm{H}), 8.08 \sim$ $8.07(\mathrm{~m}, 1 \mathrm{H}), 7.99$ (d, $J=8.0 \mathrm{~Hz}, 1 \mathrm{H}), 7.89$ (t, $J=9.0 \mathrm{~Hz}$, 2H), $7.62(\mathrm{t}, J=7.5 \mathrm{~Hz}, 1 \mathrm{H}), 7.54(\mathrm{t}, J=7.5 \mathrm{~Hz}, 1 \mathrm{H})$, $4.77 \sim 4.72(\mathrm{~m}, 2 \mathrm{H}), 3.73(\mathrm{~d}, J=22.7 \mathrm{~Hz}, 2 \mathrm{H}), 1.29(\mathrm{~d}, J=$ $2.2 \mathrm{~Hz}, 6 \mathrm{H}$, overlap), 1.26 (d, $J=1.8 \mathrm{~Hz}, 6 \mathrm{H}$, overlap); ${ }^{13} \mathrm{C}$ NMR (100 MHz, $\left.\mathrm{CDCl}_{3}\right) \delta: 191.8\left(\mathrm{~d}, J_{\mathrm{C}-\mathrm{P}}=5.7 \mathrm{~Hz}\right), 135.6$, $134.2,132.5,131.6,129.7,128.8,128.3,127.8,126.9$, 124.2, $71.4\left(\mathrm{~d}, J_{\mathrm{C}-\mathrm{P}}=5.7 \mathrm{~Hz}\right), 39.8\left(\mathrm{~d}, J_{\mathrm{C}-\mathrm{P}}=130.0 \mathrm{~Hz}\right)$, $23.8\left(\mathrm{~d}, J_{\mathrm{C}-\mathrm{P}}=3.4 \mathrm{~Hz}\right), 23.9\left(\mathrm{~d}, J_{\mathrm{C}-\mathrm{P}}=4.6 \mathrm{~Hz}\right)$; HRMS calcd for $\mathrm{C}_{18} \mathrm{H}_{24} \mathrm{O}_{4} \mathrm{P}(\mathrm{M}+\mathrm{H})^{+}$335.1412, found 335.1420.

2-氧代辛基膦酸二异丙酯 $(\mathbf{2} \mathbf{p})^{[40]}$ : 淡黄色油状物. 
${ }^{1} \mathrm{H}$ NMR (400 MHz, $\left.\mathrm{CDCl}_{3}\right) \delta: 4.74 \sim 4.69(\mathrm{~m}, 2 \mathrm{H}), 3.03$ (d, $J=22.7 \mathrm{~Hz}, 2 \mathrm{H}), 2.62$ (t, $J=7.3 \mathrm{~Hz}, 2 \mathrm{H}), 1.59 \sim 1.56$ (m, 2H), 1.35 (d, $J=2.9 \mathrm{~Hz}, 6 \mathrm{H}$, overlap), 1.32 (d, $J=2.9$ $\mathrm{Hz}, 6 \mathrm{H}$, overlap), $1.32 \sim 1.29(\mathrm{~m}, 6 \mathrm{H}), 0.88(\mathrm{t}, J=6.8 \mathrm{~Hz}$, $3 \mathrm{H}) ;{ }^{13} \mathrm{C}$ NMR $\left(100 \mathrm{MHz}, \mathrm{CDCl}_{3}\right) \delta: 202.2\left(\mathrm{~d}, J_{\mathrm{C}-\mathrm{P}}=5.7\right.$ $\mathrm{Hz}), 71.2\left(\mathrm{~d}, J_{\mathrm{C}-\mathrm{P}}=6.8 \mathrm{~Hz}\right), 43.8,43.7\left(\mathrm{~d}, J_{\mathrm{C}-\mathrm{P}}=126.6 \mathrm{~Hz}\right)$, $31.5,28.7,23.8\left(\mathrm{~d}, J_{\mathrm{C}-\mathrm{P}}=3.4 \mathrm{~Hz}\right), 23.9\left(\mathrm{~d}, J_{\mathrm{C}-\mathrm{P}}=4.6 \mathrm{~Hz}\right)$, 23.5, 22.6, 14.1; HRMS calcd for $\mathrm{C}_{14} \mathrm{H}_{29} \mathrm{O}_{4} \mathrm{NaP}(\mathrm{M}+\mathrm{Na})^{+}$ 315.1701 , found 315.1703 .

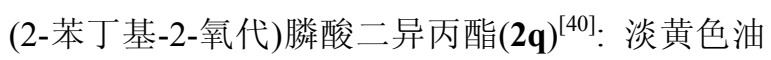
状物. ${ }^{1} \mathrm{H}$ NMR (400 MHz, $\left.\mathrm{CDCl}_{3}\right) \delta: 7.18 \sim 7.14(\mathrm{~m}, 2 \mathrm{H})$, $7.13 \sim 7.07$ (m, 3H), 3.97 (app septet, $J=7.1 \mathrm{~Hz}, 4 \mathrm{H}$ ), $3.15 \sim 3.03(\mathrm{~m}, 2 \mathrm{H}), 2.85 \sim 2.73(\mathrm{~m}, 3 \mathrm{H}), 1.25(\mathrm{~d}, J=7.1$ $\mathrm{Hz}, 3 \mathrm{H}), 1.22 \sim 1.17(\mathrm{~m}, 6 \mathrm{H}) ;{ }^{13} \mathrm{C}$ NMR $(100 \mathrm{MHz}$, $\left.\mathrm{CDCl}_{3}\right) \delta: 204.7\left(\mathrm{~d}, J_{\mathrm{C}-\mathrm{P}}=3.9 \mathrm{~Hz}\right), 140.9,128.4,128.2$, 126.0, $62.4\left(\mathrm{~d}, J_{\mathrm{C}-\mathrm{P}}=6.6 \mathrm{~Hz}\right), 62.4\left(\mathrm{~d}, J_{\mathrm{C}-\mathrm{P}}=6.8 \mathrm{~Hz}\right), 46.8$ $\left(\mathrm{d}, J_{\mathrm{C}-\mathrm{P}}=126.6 \mathrm{~Hz}\right), 44.6,29.6,16.2\left(\mathrm{~d}, J_{\mathrm{C}-\mathrm{P}}=6.8 \mathrm{~Hz}\right)$, $10.9\left(\mathrm{~d}, J_{\mathrm{C}-\mathrm{P}}=6.5 \mathrm{~Hz}\right)$; HRMS calcd for $\mathrm{C}_{15} \mathrm{H}_{24} \mathrm{O}_{4} \mathrm{P}(\mathrm{M}+$ H) ${ }^{+}$299.1412, found, 299.1414 .

1-氯-4-氧代戊基膦酸二异丙酯 $(2 \mathbf{r})^{[40]}$ : 淡黄色油状 物. ${ }^{1} \mathrm{H}$ NMR (400 MHz, $\left.\mathrm{CDCl}_{3}\right) \delta: 4.81 \sim 4.68(\mathrm{~m}, 2 \mathrm{H})$, 3.56 (t, $J=6.3 \mathrm{~Hz}, 2 \mathrm{H}), 3.04(\mathrm{~d}, J=22.9 \mathrm{~Hz}, 2 \mathrm{H}), 2.82(\mathrm{t}$, $J=6.9 \mathrm{~Hz}, 2 \mathrm{H}$ ), 2.04 (tt, apparent quint., $J=6.6,6.5 \mathrm{~Hz}$, $2 \mathrm{H}$ ), 1.33 (t, $J=6.2 \mathrm{~Hz}, 6 \mathrm{H}$, overlap), 1.32 (t, $J=6.2 \mathrm{~Hz}$, $6 \mathrm{H}$, overlap); ${ }^{13} \mathrm{C}$ NMR $\left(100 \mathrm{MHz}, \mathrm{CDCl}_{3}\right) \delta: 201.2(\mathrm{~d}$, $\left.J_{\mathrm{C}-\mathrm{P}}=5.6 \mathrm{~Hz}\right), 71.7\left(\mathrm{~d}, J_{\mathrm{C}-\mathrm{P}}=6.7 \mathrm{~Hz}\right), 44.3,44.2\left(\mathrm{~d}, J_{\mathrm{C}-\mathrm{P}}=\right.$ $127.7 \mathrm{~Hz}), 40.7,26.4,16.2\left(\mathrm{~d}, J_{\mathrm{C}-\mathrm{P}}=3.9 \mathrm{~Hz}\right), 24.1(\mathrm{~d}$, $\left.J_{\mathrm{C}-\mathrm{P}}=5.0 \mathrm{~Hz}\right)$; HRMS calcd for $\mathrm{C}_{11} \mathrm{H}_{22} \mathrm{ClO}_{4} \mathrm{P}(\mathrm{M}+\mathrm{Na})^{+}$ 307.0840, found 307.0842 .

1-腈基-4-氧代戊基膦酸二乙酯 $(2 \mathbf{s})^{[41]}$ : 淡黄色油状 物. ${ }^{1} \mathrm{H}$ NMR (400 MHz, $\left.\mathrm{CDCl}_{3}\right) \delta: 4.78 \sim 4.61(\mathrm{~m}, 2 \mathrm{H})$, $3.02(\mathrm{~d}, J=22.9 \mathrm{~Hz}, 2 \mathrm{H}), 2.81$ (t, $J=6.8 \mathrm{~Hz}, 2 \mathrm{H}), 2.39$ (t, $J=7.1 \mathrm{~Hz}, 2 \mathrm{H}$ ), 1.93 (tt, apparent quint, $J=6.9,6.9 \mathrm{~Hz}$, 2H), 1.34 (t, $J=6.2 \mathrm{~Hz}, 6 \mathrm{H}$, overlap), 1.32 (t, $J=6.2 \mathrm{~Hz}$, $6 \mathrm{H}$, overlap); ${ }^{13} \mathrm{C}$ NMR (100 MHz, $\left.\mathrm{CDCl}_{3}\right) \delta: 200.4$ (d, $\left.J_{\mathrm{C}-\mathrm{P}}=5.6 \mathrm{~Hz}\right), 119.2,71.8\left(\mathrm{~d}, J_{\mathrm{C}-\mathrm{P}}=6.7 \mathrm{~Hz}\right), 44.3\left(\mathrm{~d}, J_{\mathrm{C}-\mathrm{P}}=\right.$ $127.4 \mathrm{~Hz}), 41.6,24.1\left(\mathrm{~d}, J_{\mathrm{C}-\mathrm{P}}=4.0 \mathrm{~Hz}\right), 23.8\left(\mathrm{~d}, J_{\mathrm{C}-\mathrm{P}}=4.9\right.$ $\mathrm{Hz}$ ); HRMS calcd for $\mathrm{C}_{12} \mathrm{H}_{22} \mathrm{NO}_{4} \mathrm{P}(\mathrm{M}+\mathrm{Na})^{+} 298.1173$, found 298.1184 .

2-氧代-环已烯基乙基膦酸二乙酯(2t) ${ }^{[40]}$ : 淡黄色油 状物. ${ }^{1} \mathrm{H}$ NMR (400 MHz, $\left.\mathrm{CDCl}_{3}\right) \delta: 7.01 \sim 6.96(\mathrm{~m}, 1 \mathrm{H})$, $4.73 \sim 4.61(\mathrm{~m}, 2 \mathrm{H}), 3.25\left(\mathrm{~d}, J_{\mathrm{H}-\mathrm{P}}=22.7 \mathrm{~Hz}, 2 \mathrm{H}\right), 2.28 \sim$ $2.22(\mathrm{~m}, 2 \mathrm{H}), 2.21 \sim 2.15(\mathrm{~m}, 2 \mathrm{H}), 1.63 \sim 1.53(\mathrm{~m}, 4 \mathrm{H}$, overlap), 1.28 (d, $J=6.2 \mathrm{~Hz}, 6 \mathrm{H}$, overlap), 1.27 (d, $J=6.2$ $\mathrm{Hz}, 6 \mathrm{H}$, overlap); ${ }^{13} \mathrm{C} \mathrm{NMR}\left(100 \mathrm{MHz}, \mathrm{CDCl}_{3}\right) \delta: 192.8$ (d,
$\left.J_{\mathrm{C}-\mathrm{P}}=6.1 \mathrm{~Hz}\right), 143.4,139.4,71.3\left(\mathrm{~d}, J_{\mathrm{C}-\mathrm{P}}=6.7 \mathrm{~Hz}\right), 38.5$ $\left(\mathrm{d}, J_{\mathrm{C}-\mathrm{P}}=131.3 \mathrm{~Hz}\right), 26.3,24.2\left(\mathrm{~d}, J_{\mathrm{C}-\mathrm{P}}=3.9 \mathrm{~Hz}\right), 23.9(\mathrm{~d}$, $\left.J_{\mathrm{C}-\mathrm{P}}=5.2 \mathrm{~Hz}\right), 23.3,21.8,21.6$; HRMS calcd for $\mathrm{C}_{14} \mathrm{H}_{25} \mathrm{O}_{4} \mathrm{P}(\mathrm{M}+\mathrm{Na})^{+}$311.1383, found 311.1388.

2-氧代-环已烷基乙基膦酸二乙酯 $(\mathbf{2 u})^{[42]}$ : 淡黄色油 状物. ${ }^{1} \mathrm{H}$ NMR $\left(400 \mathrm{MHz}, \mathrm{CDCl}_{3}\right) \delta: 4.12 \sim 4.05(\mathrm{~m}, 4 \mathrm{H})$, $3.07\left(\mathrm{~d}, J_{\mathrm{H}-\mathrm{P}}=22.4 \mathrm{~Hz}, 2 \mathrm{H}\right), 2.57 \sim 2.51(\mathrm{~m}, 1 \mathrm{H}), 1.85 \sim$ $1.83(\mathrm{~m}, 2 \mathrm{H}), 1.73 \sim 1.70(\mathrm{~m}, 2 \mathrm{H}), 1.62 \sim 1.58(\mathrm{~m}, 1 \mathrm{H})$, $1.28(\mathrm{t}, J=7.2 \mathrm{~Hz}, 11 \mathrm{H}) ;{ }^{13} \mathrm{C}$ NMR $\left(100 \mathrm{MHz}, \mathrm{CDCl}_{3}\right) \delta$ : $205.26\left(\mathrm{~d}, J_{\mathrm{C}-\mathrm{P}}=5.8 \mathrm{~Hz}\right), 62.37\left(\mathrm{~d}, J_{\mathrm{C}-\mathrm{P}}=6.6 \mathrm{~Hz}\right), 51.28$, $40.12\left(\mathrm{~d}, J_{\mathrm{C}-\mathrm{P}}=127.0 \mathrm{~Hz}\right), 28.06,25.61,25.36,16.19(\mathrm{~d}$, $\left.J_{\mathrm{C}-\mathrm{P}}=6.6 \mathrm{~Hz}\right)$; HRMS calcd for $\mathrm{C}_{12} \mathrm{H}_{23} \mathrm{O}_{4} \mathrm{P}(\mathrm{M}+\mathrm{H})^{+}$ 262.1336 , found 262.1331 .

(2-苯乙基-2-氧代)膦酸二乙酯 $(\mathbf{2 v})^{[41]}$ : 淡黄色油状 物. ${ }^{1} \mathrm{H}$ NMR $\left(400 \mathrm{MHz}, \mathrm{CDCl}_{3}\right) \delta: 8.01(\mathrm{~d}, J=8.1 \mathrm{~Hz}$, $2 \mathrm{H}), 7.59$ (t, $J=7.4 \mathrm{~Hz}, 1 \mathrm{H}), 7.48(\mathrm{t}, J=7.7 \mathrm{~Hz}, 2 \mathrm{H})$, $4.18 \sim 4.12(\mathrm{~m}, 4 \mathrm{H}), 3.64$ (d, $J=22.7 \mathrm{~Hz}, 2 \mathrm{H}), 1.27$ (t, $J=$ $7.1 \mathrm{~Hz}, 6 \mathrm{H}) ;{ }^{13} \mathrm{C}$ NMR $\left(100 \mathrm{MHz}, \mathrm{CDCl}_{3}\right) \delta: 191.9(\mathrm{~d}$, $\left.J_{\mathrm{C}-\mathrm{P}}=6.8 \mathrm{~Hz}\right), 136.7,133.5,129.1,128.7,62.7\left(\mathrm{~d}, J_{\mathrm{C}-\mathrm{P}}=\right.$ $5.7 \mathrm{~Hz}), 38.6\left(\mathrm{~d}, J_{\mathrm{C}-\mathrm{P}}=128.9 \mathrm{~Hz}\right), 16.1\left(\mathrm{~d}, J_{\mathrm{C}-\mathrm{P}}=5.7 \mathrm{~Hz}\right)$; HRMS calcd for $\mathrm{C}_{12} \mathrm{H}_{18} \mathrm{O}_{4} \mathrm{P}(\mathrm{M}+\mathrm{H})^{+}$257.0943, found 257.0947.

(2-苯乙基-2-氧代)膦酸二甲酯 $(\mathbf{2 w})^{[40]}$ : 淡黄色油状 物. ${ }^{1} \mathrm{H}$ NMR $\left(400 \mathrm{MHz}, \mathrm{CDCl}_{3}\right) \delta: 8.01(\mathrm{~d}, J=8.6 \mathrm{~Hz}$, 2H), 7.58 (t, $J=7.4 \mathrm{~Hz}, 1 \mathrm{H}), 7.47$ (t, $J=7.4 \mathrm{~Hz}, 2 \mathrm{H}), 3.76$ (d, $J=11.2 \mathrm{~Hz}, 6 \mathrm{H}), 3.65(\mathrm{~d}, J=22.6 \mathrm{~Hz}, 2 \mathrm{H}) ;{ }^{13} \mathrm{C}$ NMR $\left(100 \mathrm{MHz}, \mathrm{CDCl}_{3}\right) \delta: 191.8\left(\mathrm{~d}, J_{\mathrm{C}-\mathrm{P}}=6.6 \mathrm{~Hz}\right), 136.4(\mathrm{~d}$, $\left.J_{\mathrm{C}-\mathrm{P}}=2.3 \mathrm{~Hz}\right), 133.9,129.1,128.8,53.2\left(\mathrm{~d}, J_{\mathrm{C}-\mathrm{P}}=6.5 \mathrm{~Hz}\right)$, $37.6\left(\mathrm{~d}, J_{\mathrm{C}-\mathrm{P}}=131.2 \mathrm{~Hz}\right)$; HRMS calcd for $\mathrm{C}_{10} \mathrm{H}_{14} \mathrm{O}_{4} \mathrm{P}$ $(\mathrm{M}+\mathrm{H})^{+} 229.0630$, found 229.0635 .

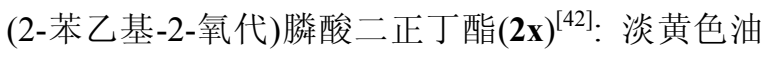
状物. ${ }^{1} \mathrm{H}$ NMR $\left(400 \mathrm{MHz}, \mathrm{CDCl}_{3}\right) \delta: 8.02(\mathrm{~d}, J=7.7 \mathrm{~Hz}$, $2 \mathrm{H}), 7.59(\mathrm{t}, J=7.5 \mathrm{~Hz}, 1 \mathrm{H}), 7.48(\mathrm{t}, J=7.7 \mathrm{~Hz}, 2 \mathrm{H})$, $4.11 \sim 4.03(\mathrm{~m}, 4 \mathrm{H}), 3.62(\mathrm{~d}, J=22.7 \mathrm{~Hz}, 2 \mathrm{H}), 1.63 \sim 1.57$ (m, 4H), $1.35 \sim 1.28(\mathrm{~m}, 4 \mathrm{H}), 0.89(\mathrm{t}, J=7.5 \mathrm{~Hz}, 6 \mathrm{H}) ;{ }^{13} \mathrm{C}$ NMR $\left(100 \mathrm{MHz}, \mathrm{CDCl}_{3}\right) \delta: 191.8,136.7,133.5,129.1$, $128.7,66.2\left(\mathrm{~d}, J_{\mathrm{C}-\mathrm{P}}=6.8 \mathrm{~Hz}\right), 38.5\left(\mathrm{~d}, J_{\mathrm{C}-\mathrm{P}}=128.9 \mathrm{~Hz}\right)$, $32.2\left(\mathrm{~d}, J_{\mathrm{C}-\mathrm{P}}=5.7 \mathrm{~Hz}\right), 18.5,13.6$; HRMS calcd for $\mathrm{C}_{16} \mathrm{H}_{26} \mathrm{O}_{4} \mathrm{P}(\mathrm{M}+\mathrm{H})^{+}$313.1569, found 313.1573.

(2-苯乙基-2-氧代)膦酸二芐酯 $(2 \mathbf{y})^{[40]}$ : 淡黄色油状 物. ${ }^{1} \mathrm{H}$ NMR $\left(400 \mathrm{MHz}, \mathrm{CDCl}_{3}\right) \delta: 7.86 \sim 7.83(\mathrm{~m}, 2 \mathrm{H})$, $7.46 \sim 7.42(\mathrm{~m}, 1 \mathrm{H}), 7.34 \sim 7.31(\mathrm{~m}, 2 \mathrm{H}), 7.23 \sim 7.17(\mathrm{~m}$, $10 \mathrm{H}), 5.01 \sim 4.90(\mathrm{~m}, 4 \mathrm{H}), 3.54\left(\mathrm{~d}, J_{\mathrm{H}-\mathrm{P}}=22.8 \mathrm{~Hz}, 2 \mathrm{H}\right)$; ${ }^{13} \mathrm{C}$ NMR $\left(100 \mathrm{MHz}, \mathrm{CDCl}_{3}\right) \delta: 191.25\left(\mathrm{~d}, J_{\mathrm{C}-\mathrm{P}}=6.6 \mathrm{~Hz}\right)$, $136.31\left(\mathrm{~d}, J_{\mathrm{C}-\mathrm{P}}=2.2 \mathrm{~Hz}\right), 135.75\left(\mathrm{~d}, J_{\mathrm{C}-\mathrm{P}}=5.8 \mathrm{~Hz}\right), 133.55$, 
$128.88,128.48,128.42,128.34,127.87,67.91\left(\mathrm{~d}, J_{\mathrm{C}-\mathrm{P}}=6.6\right.$ $\mathrm{Hz}), 38.50\left(\mathrm{~d}, J_{\mathrm{C}-\mathrm{P}}=130.5 \mathrm{~Hz}\right)$; HRMS calcd for $\mathrm{C}_{22} \mathrm{H}_{21} \mathrm{O}_{4} \mathrm{P}(\mathrm{M}+\mathrm{H})^{+}$380.1179, found 380.1172 .

辅助材料(Supporting Information) 化合物 $\mathbf{2 a} \sim 2 \mathrm{y}$ 的 氢谱. 这些材料可以免费从本刊网站(http://sioc-journal. $\mathrm{cn} /)$ 上下载.

\section{References}

[1] Beller, M.; Seayad, J.; Tillack, A.; Jiao, H. Angew. Chem., Int. Ed. 2004, 43, 3368.

[2] Hintermann, L.; Labonne, A. Synthesis 2007, 1121.

[3] Kutscheroff, M. Chem. Ber. 1881, 14, 1540.

[4] Suzuki, T.; Tokunaga, M.; Wakatsuki, Y. Org. Lett. 2001, 3, 735.

[5] Tokunaga, M.; Suzuki, T.; Koga, N.; Fukushima, T.; Horiuchi, A.; Wakatsuki, Y. J. Am. Chem. Soc. 2001, 123, 11917.

[6] Baidossi, W.; Lahav, M.; Blum, J. J. Org. Chem. 1997, 62, 669.

[7] Trentin, F.; Chapman, A. M.; Scarso, A.; Sgarbossa, P.; Michelin, R. A.; Strukul, G. D.; Wass, F. Adv. Synth. Catal. 2012, 354, 1095.

[8] Wu, X.-F.; Bezier, D.; Darcel, C. Adv. Synth. Catal. 2009, 351, 367.

[9] Cabrero-Antonino, J. R.; Leyva-Pérez, A.; Corma, A. Chem.-Eur. J. 2012, 18, 11107.

[10] Park, J.; Yeon, J.; Lee, P. H.; Lee, K. Tetrahedron Lett. 2013, 54, 4414.

[11] Das, R.; Chakraborty, D. Appl. Organomet. Chem. 2012, 26, 722.

[12] Thuong, M. B. T.; Mann, A.; Wagner, A. Chem. Commun. 2012, $48,434$.

[13] Chen, Z.-W.; Ye, D.-N.; Qian, Y.-P.; Ye, M.; Liu, L.-X. Tetrahedron 2013, 69, 6116.

[14] Tachinami, T.; Nishimura, T.; Ushimaru, R.; Noyori, R.; Naka, H. J. Am. Chem. Soc. 2012, 135, 50.

[15] Wong, W.-L.; Ho, K.-P.; Lee, L. Y. S.; Lam, K.-M.; Zhou, Z.-Y.; Chan, T. H.; Wong, K.-Y. ACS Catal. 2011, 1, 116.

[16] Ackermann, L.; Kaspar, L. T. J. Org. Chem. 2007, 72, 6149.

[17] Wang, S.; Zhang, G.; Zhang, L. Synlett 2010, 692.

[18] Huang, H.; Zhou, Y.; Liu, H. Beilstein J. Org. Chem. 2011, 7, 897.

[19] Xiao, J.; Li, X. Angew. Chem., Int. Ed. 2011, 50, 7226.

[20] Lu, B.-L.; Dai, L.; Shi, M. Chem. Soc. Rev. 2012, 41, 3318 (in Chinese).

[21] Zhang, Y.; Luo, S.; Zhu, C. J. Chin. J. Org. Chem. 2012, 32, 2073 (in Chinese).

(张艳, 罗莎, 朱成建, 有机化学, 2012, 32, 2073.)

[22] Fukuda, Y.; Utimoto, K. J. Org. Chem. 1991, 56, 3729.
[23] Mizushima, E.; Sato, K.; Hayashi, T.; Tanaka, M. Angew. Chem., Int. Ed. 2002, 41, 4563.

[24] Casado, R.; Contel, M.; Laguna, M.; Romero, P.; Sanz, S. J. Am. Chem. Soc. 2003, 125, 11925.

[25] Roembke, P.; Schmidbaur, H.; Cronje, S.; Raubenheimer, H. J. Mol. Catal. A: Chem. 2004, 212, 35.

[26] Marion, N.; Ramón, R. n. S.; Nolan, S. P. J. Am. Chem. Soc. 2009, $131,448$.

[27] Ghosh, N.; Nayak, S.; Sahoo, A. K. J. Org. Chem. 2010, 76, 500.

[28] Czégéni, C. E.; Papp, G.; Kathó, Á.; Joó, F. J. Mol. Catal. A: Chem. 2011, 340, 1 .

[29] Nun, P.; Ramón, R. S.; Gaillard, S.; Nolan, S. P. J. Organomet. Chem. 2011, 696, 7 .

[30] Xu, X.; Kim, S. H.; Zhang, X.; Das, A. K.; Hirao, H.; Hong, S. H. Organometallics 2012, 32, 164.

[31] Tubaro, C.; Baron, M.; Biffis, A.; Basato, M. Beilstein J. Org. Chem. 2013, 9, 246.

[32] Kondoh, A.; Yorimitsu, H.; Oshima, K. Chem. Asian J. 2010, 5, 398.

[33] Zhou, Y.; Wang, G.; Saga, Y.; Shen, R.; Goto, M.; Zhao, Y.; Han, L.-B. J. Org. Chem. 2010, 75, 7924.

[34] Li, Y.; Das, S.; Zhou, S.; Junge, K.; Beller, M. J. Am. Chem. Soc. 2012, 134, 9727

[35] Maryanoff, B. E.; Reitz, A. B. Chem. Rev. 1989, 89, 863.

[36] Pronin, S. V.; Martinez, A.; Kuznedelov, K.; Severinov, K.; Shuman, H. A.; Kozmin, S. A. J. Am. Chem. Soc. 2011, 133, 12172 .

[37] Ryglowski, A.; Kafarski, P. Tetrahedron 1996, 52, 10685.

[38] Kitamura, M.; Tokunaga, M.; Noyori, R. J. Am. Chem. Soc. 1995, 117, 2931.

[39] Chávez, M. Á.; Vargas, S.; Suárez, A.; Álvarez, E.; Pizzano, A. Adv. Synth. Catal. 2011, 353, 2775.

[40] Li, X.; Hu, G.; Luo, P.; Tang, G.; Gao, Y.; Xu, P.; Zhao, Y. Adv. Synth. Catal. 2012, 354, 2427.

[41] Wei, W.; Ji, J.-X. Angew. Chem., Int. Ed. 2011, 50, 9097.

[42] Maloney, K. M.; Chung, J. Y. L. J. Org. Chem. 2009, 74, 7574.

[43] Koprowski, M.; Szymańska, D.; Bodzioch, A.; Marciniak, B.; Różycka-Sokołowska, E.; Bałczewski, P. Tetrahedron 2009, 65, 4017.

[44] Milburn, R. R.; McRae, K.; Chan, J.; Tedrow, J.; Larsen, R.; Faul, M. Tetrahedron Lett. 2009, 50, 870.

[45] Gao, Y.; Wang, G.; Chen, L.; Xu, P.; Zhao, Y.; Zhou, Y.; Han, L.-B. J. Am. Chem. Soc. 2009, 131, 7956.

[46] Toy, A. D. F.; Walsh, E. N. In Phosphorus Chemistry in Everyday Living, American Chemical Society, Washington D. C., 1987.

[47] Essid, I.; Touil, S. ARKIVOC 2013, 4, 98. 


\section{辅助材料(Supporting Information)}

\section{金催化炔基膦酸酯水合反应合成 $\beta$-羰基膦酸酯}

刘开建* 刘宏伟王文革欧丽娟王津津胡波年*

(湖南工学院材料与化学工程系 衡阳 421002)

${ }^{1} \mathrm{H}$ NMR of $\mathbf{2} \mathbf{a} \sim \mathbf{2 y}$ 


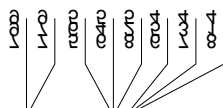

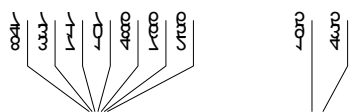<smiles>CC(C)OP(=O)(CC(=O)c1ccccc1)OC(C)C</smiles>

$2 a$
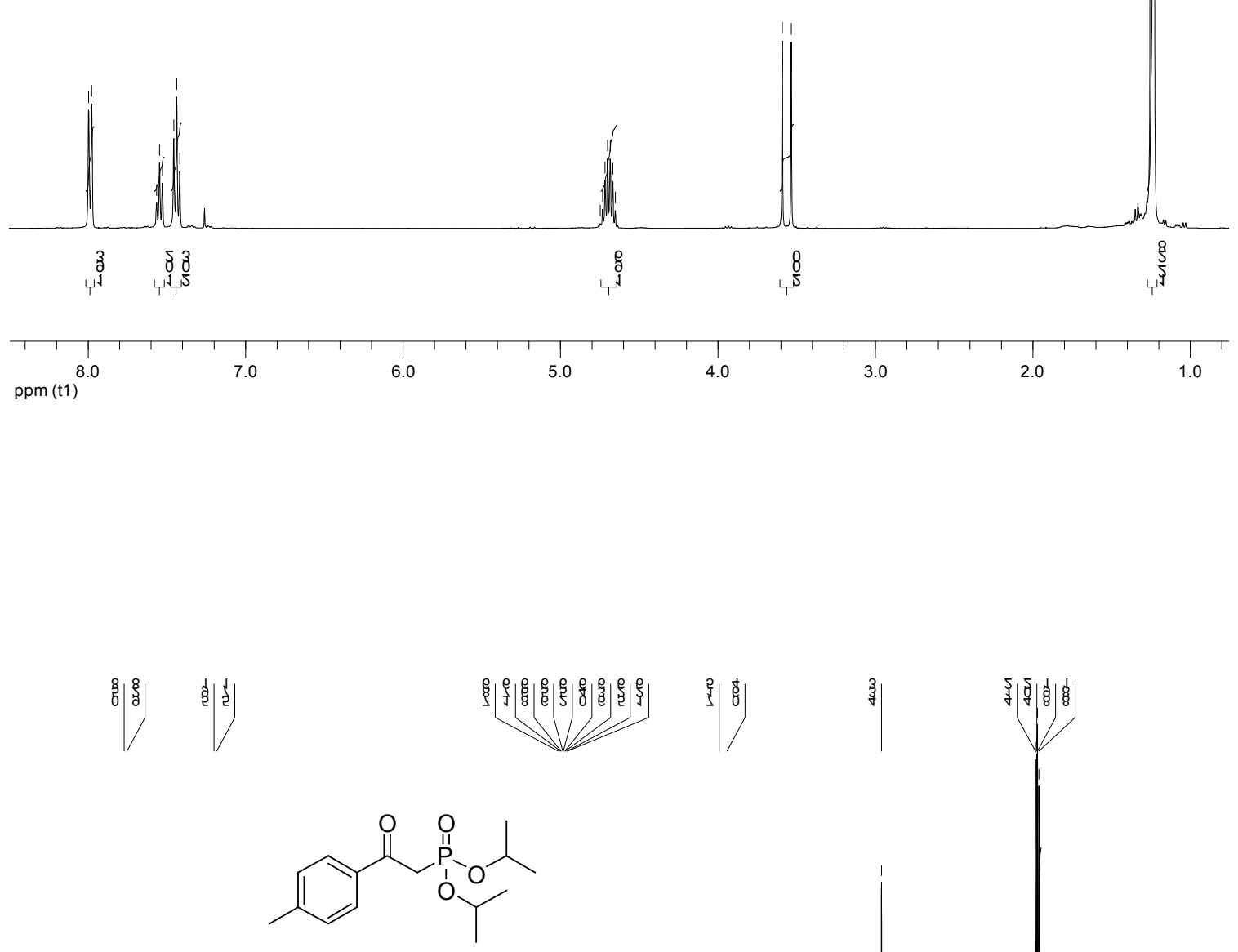

$2 b$
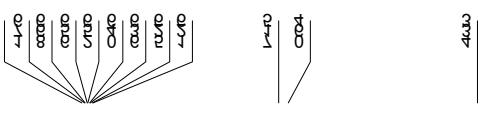

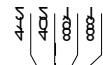
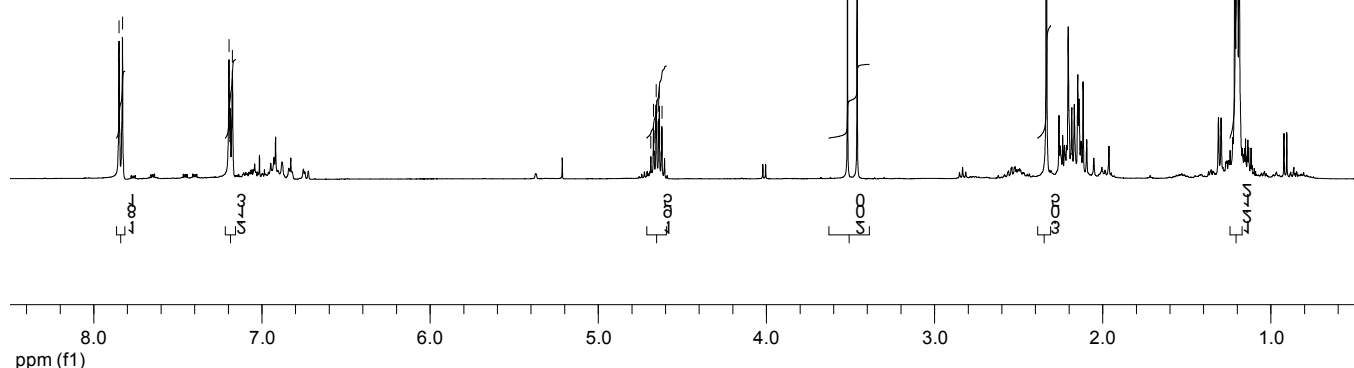


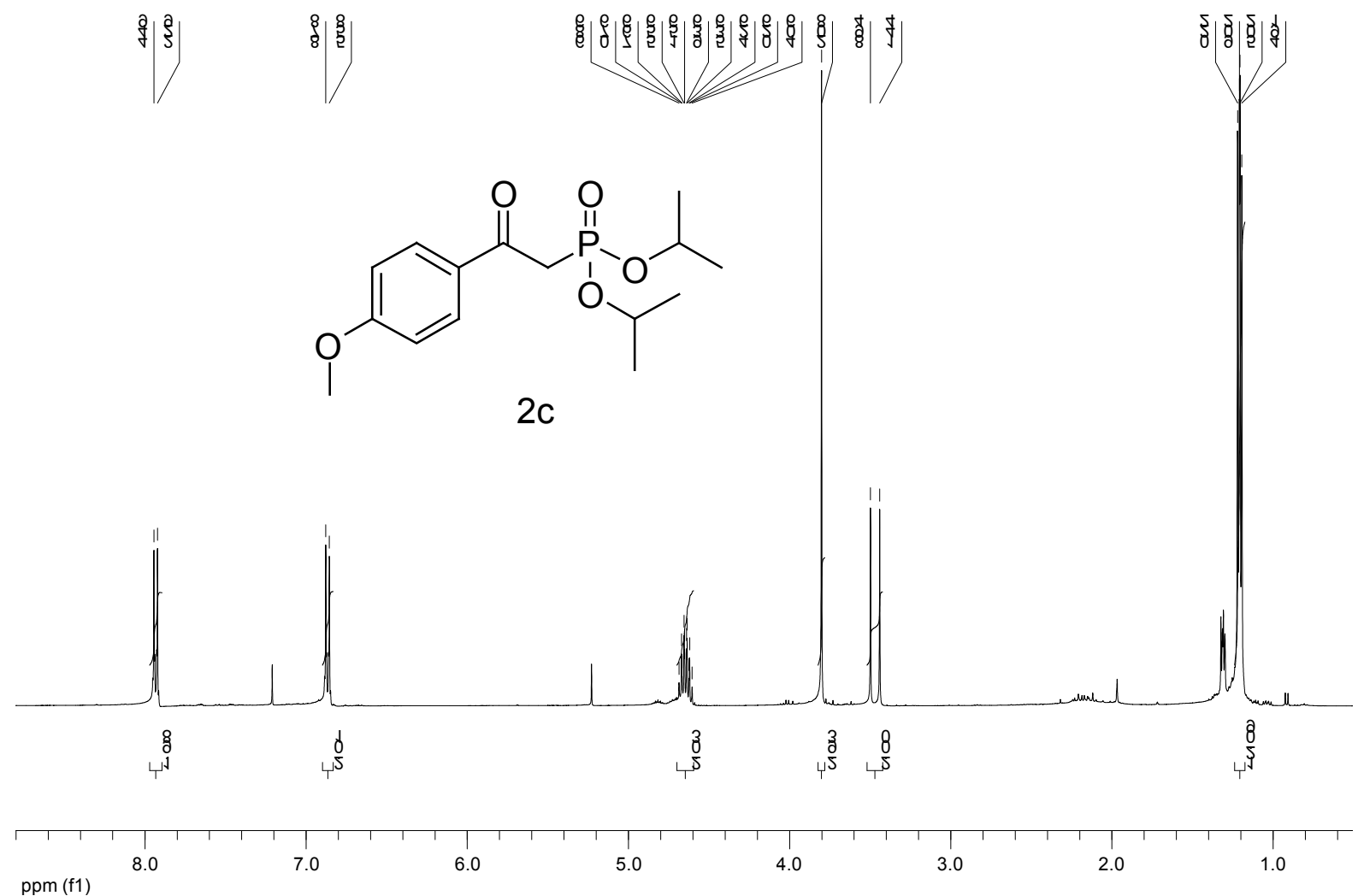

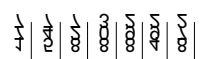

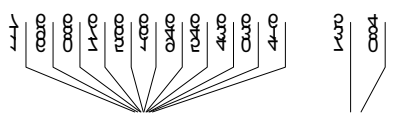

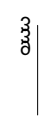

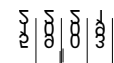<smiles>Cc1cccc(C(=O)CP(=O)(OC(C)C)OC(C)C)c1</smiles>

$2 d$

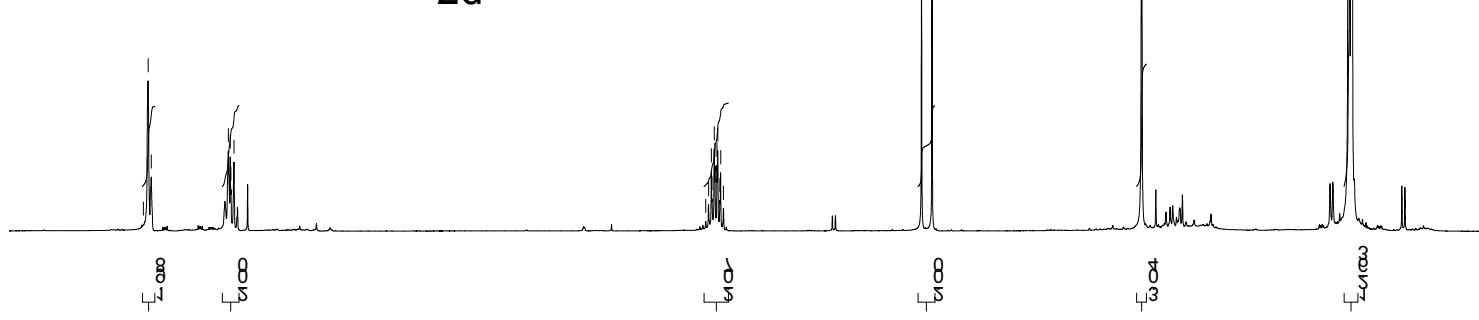

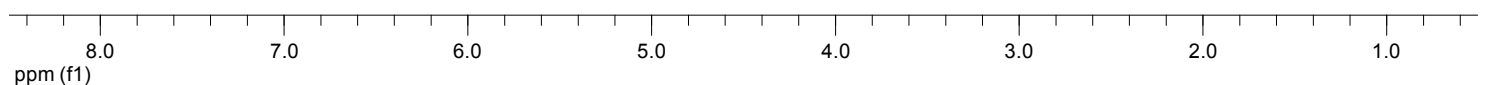


१|ํำ

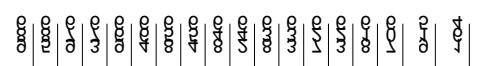

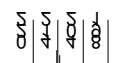<smiles>CC(C)OP(=O)(CC(=O)c1ccc(Br)cc1)OC(C)C</smiles>

$2 \mathrm{e}$
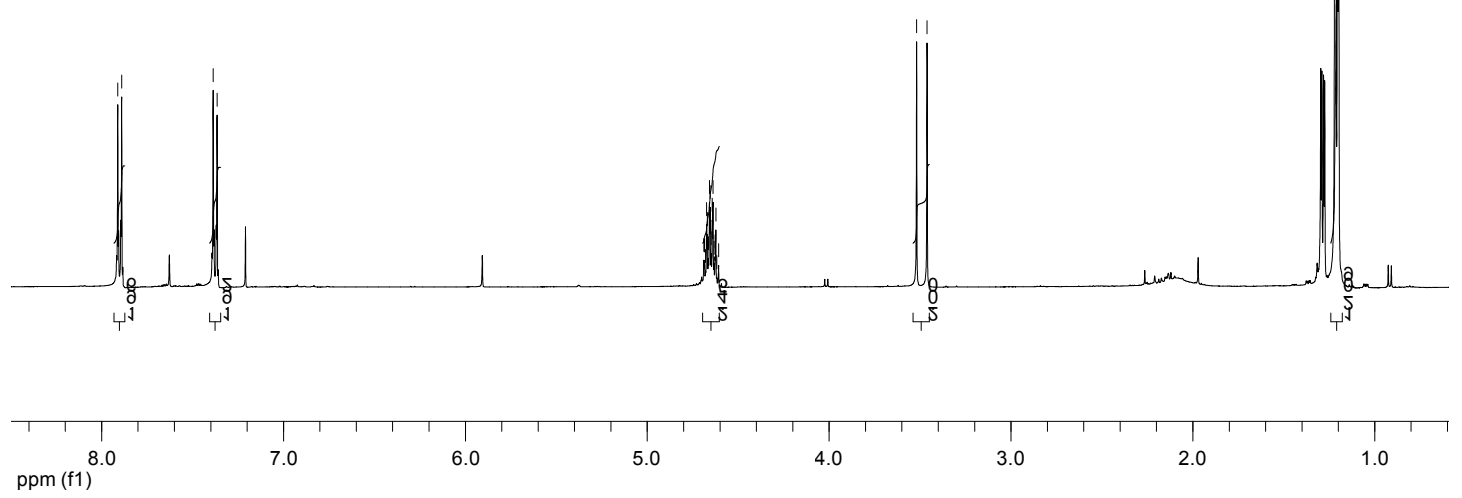

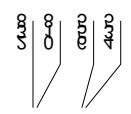

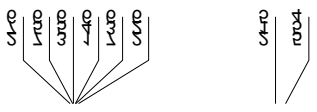

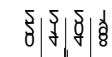<smiles>CC(C)OP(=O)(CC(=O)c1ccc(Cl)cc1)OC(C)C</smiles>

$2 f$
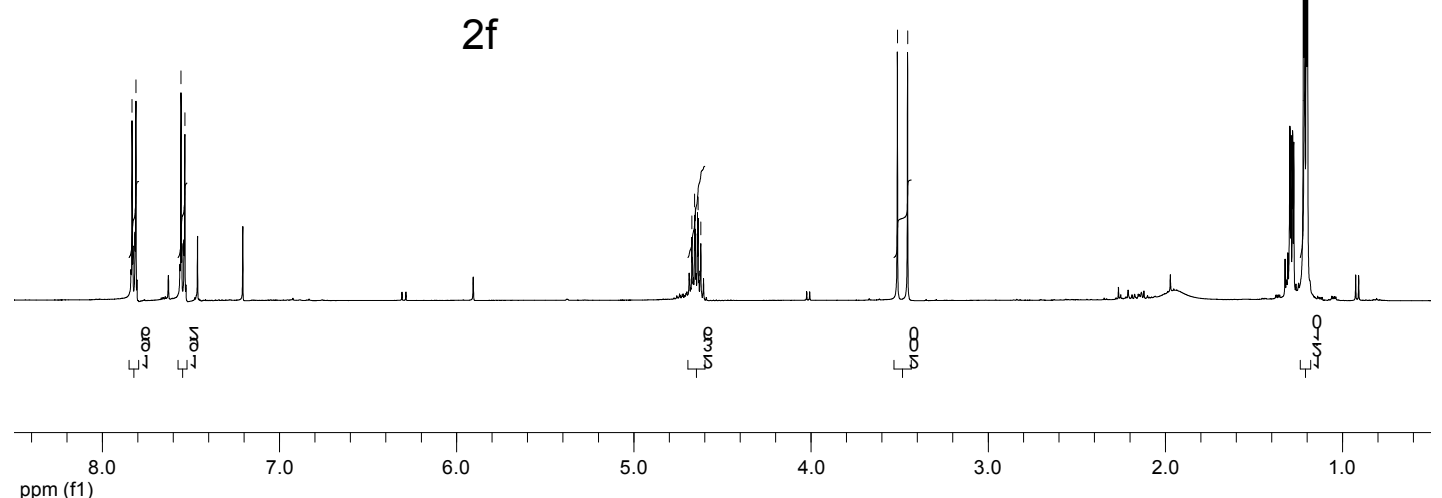
\&! ํํำ

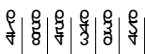

$\stackrel{?}{\text { క }}$

ร.ำ.

$\overbrace{1}^{O}$

$2 g$

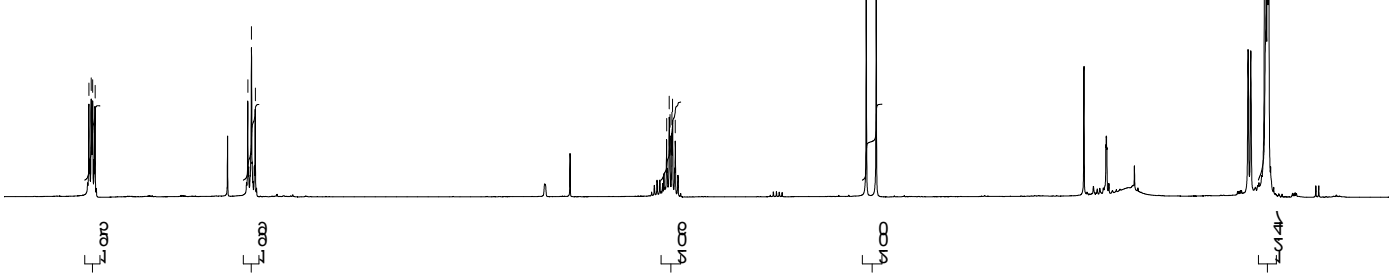

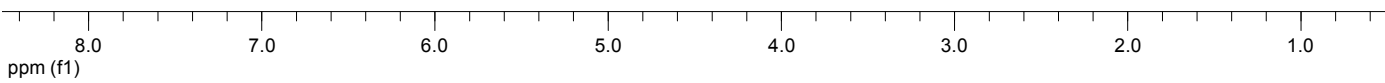




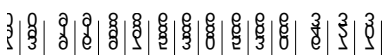

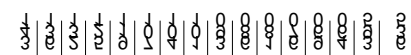

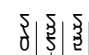<smiles>CCOP(=O)(CC(=O)c1cccc(Br)c1)OCC</smiles>

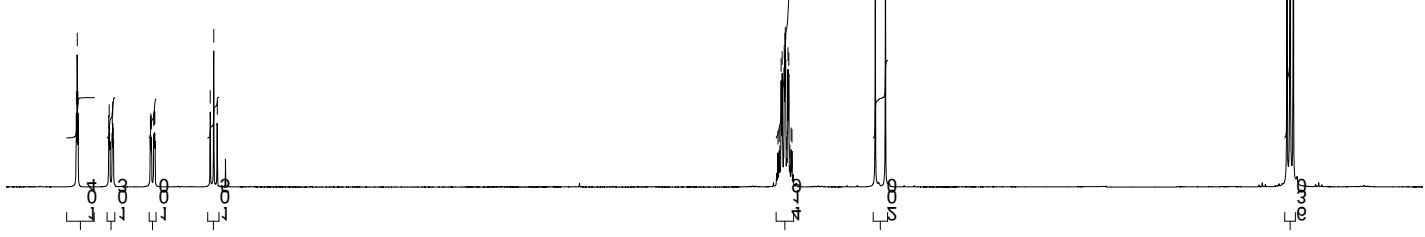

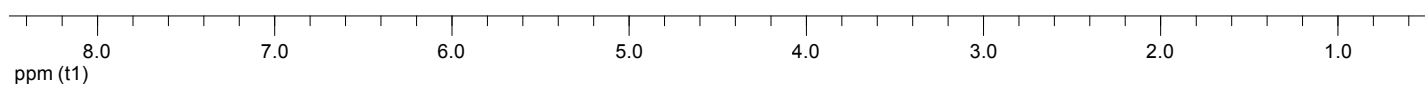

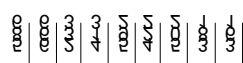

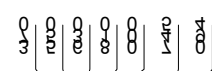<smiles>CCOP(=O)(CC(=O)c1ccccc1C)OCC</smiles>

2j

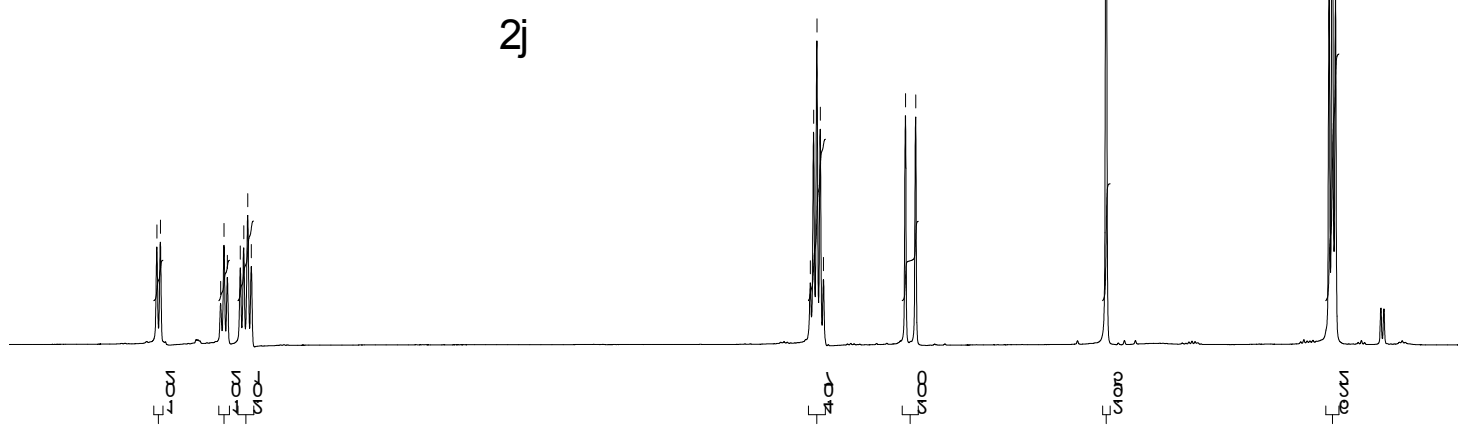

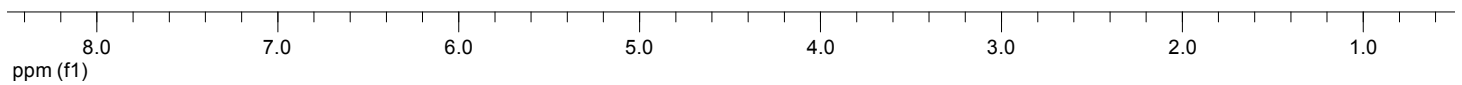




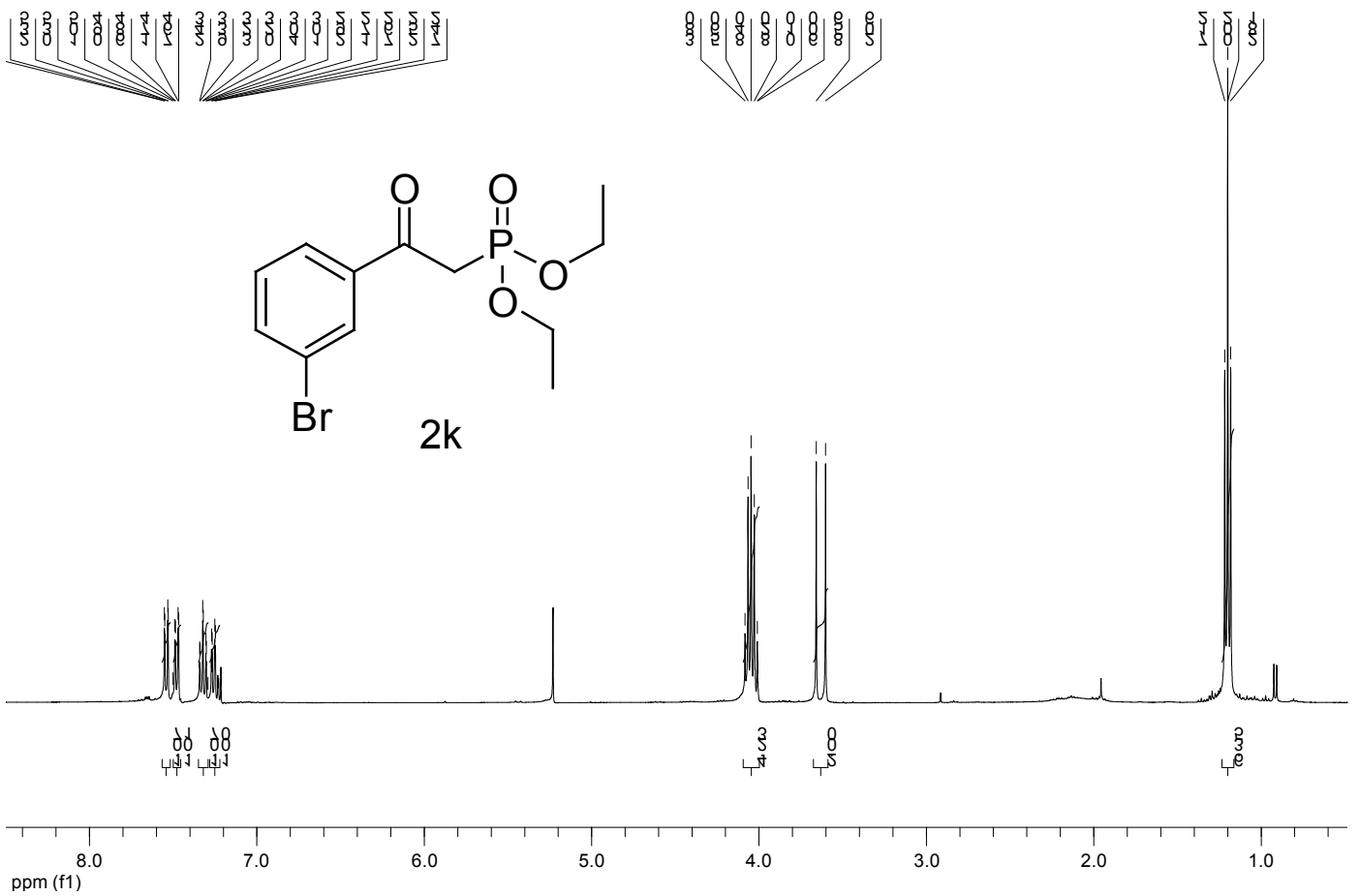

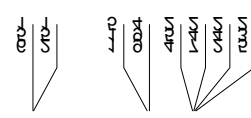

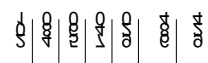

素亭孝<smiles>CCOP(=O)(CC(=O)c1ccsc1)OCC</smiles>

21

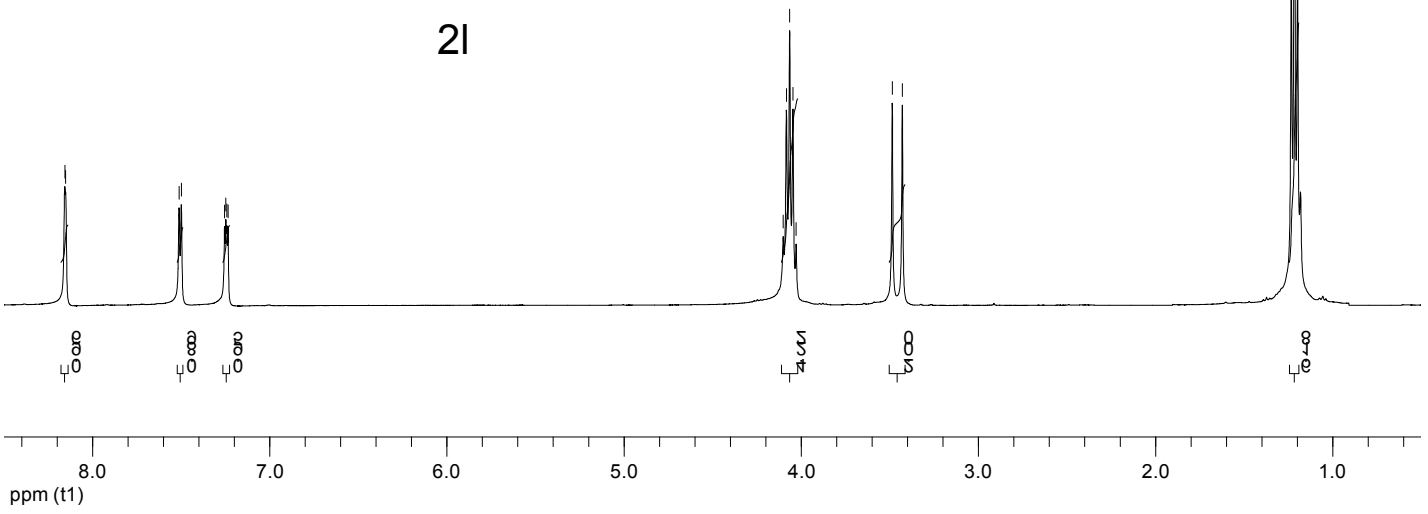




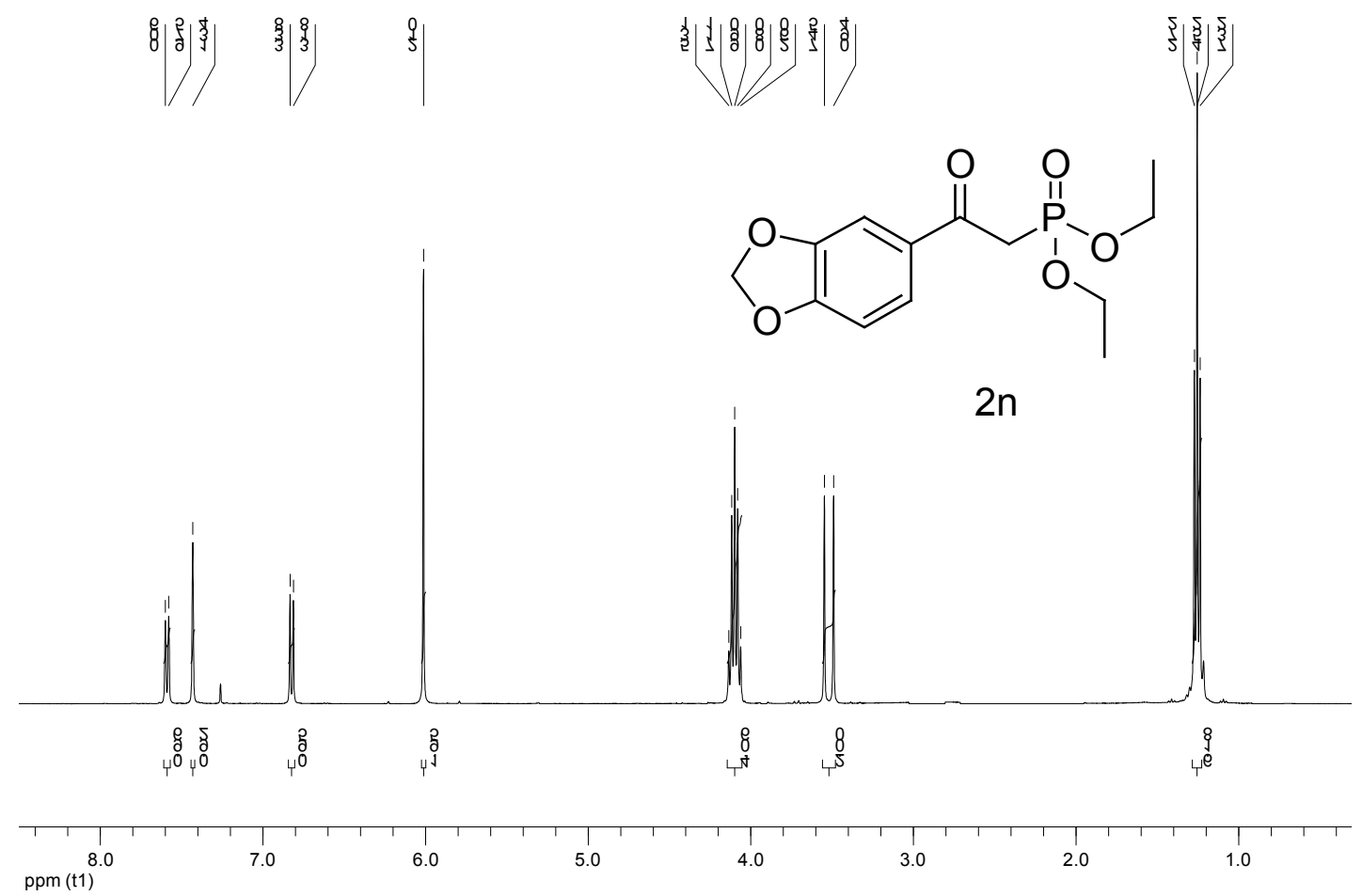




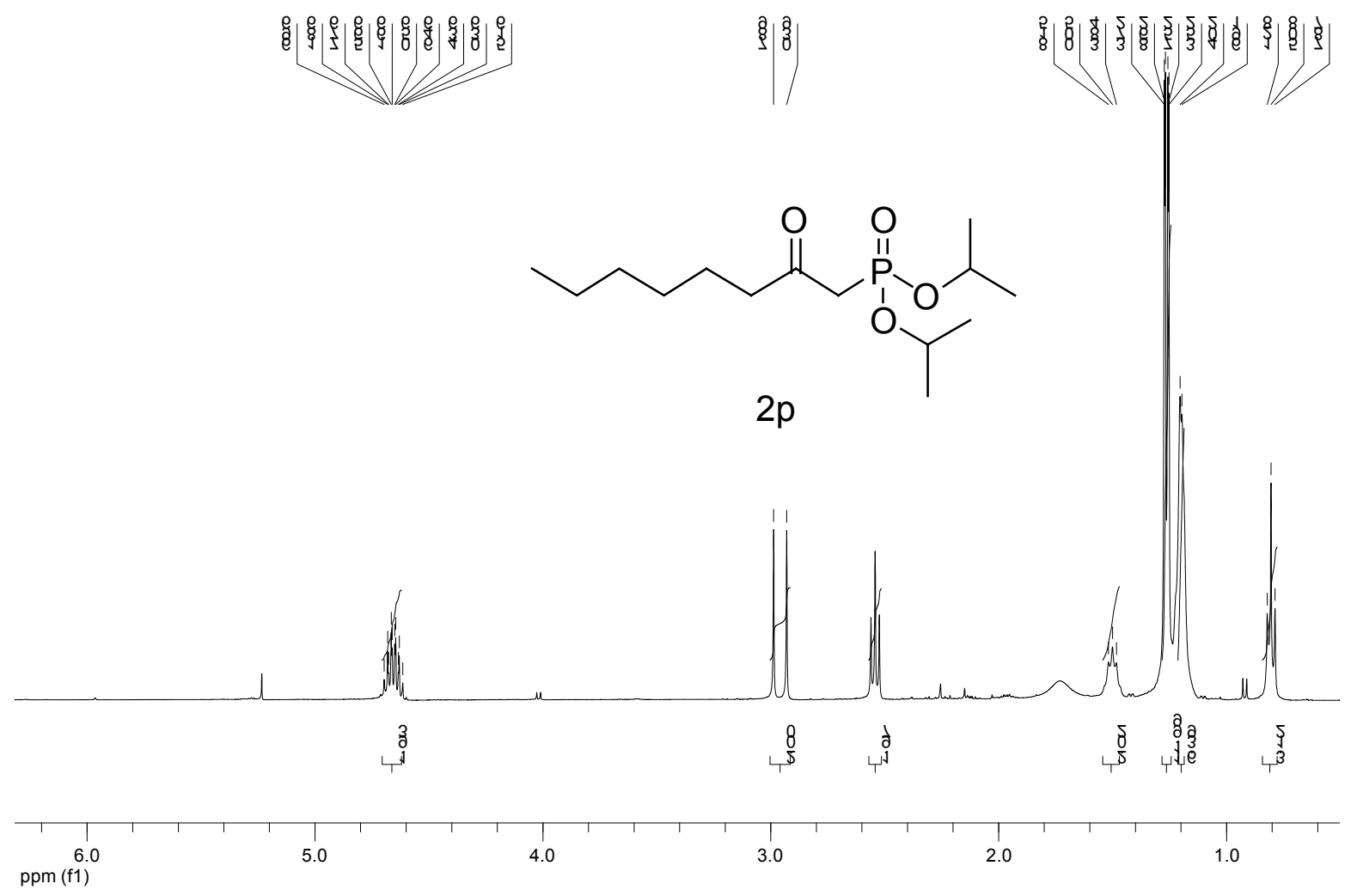




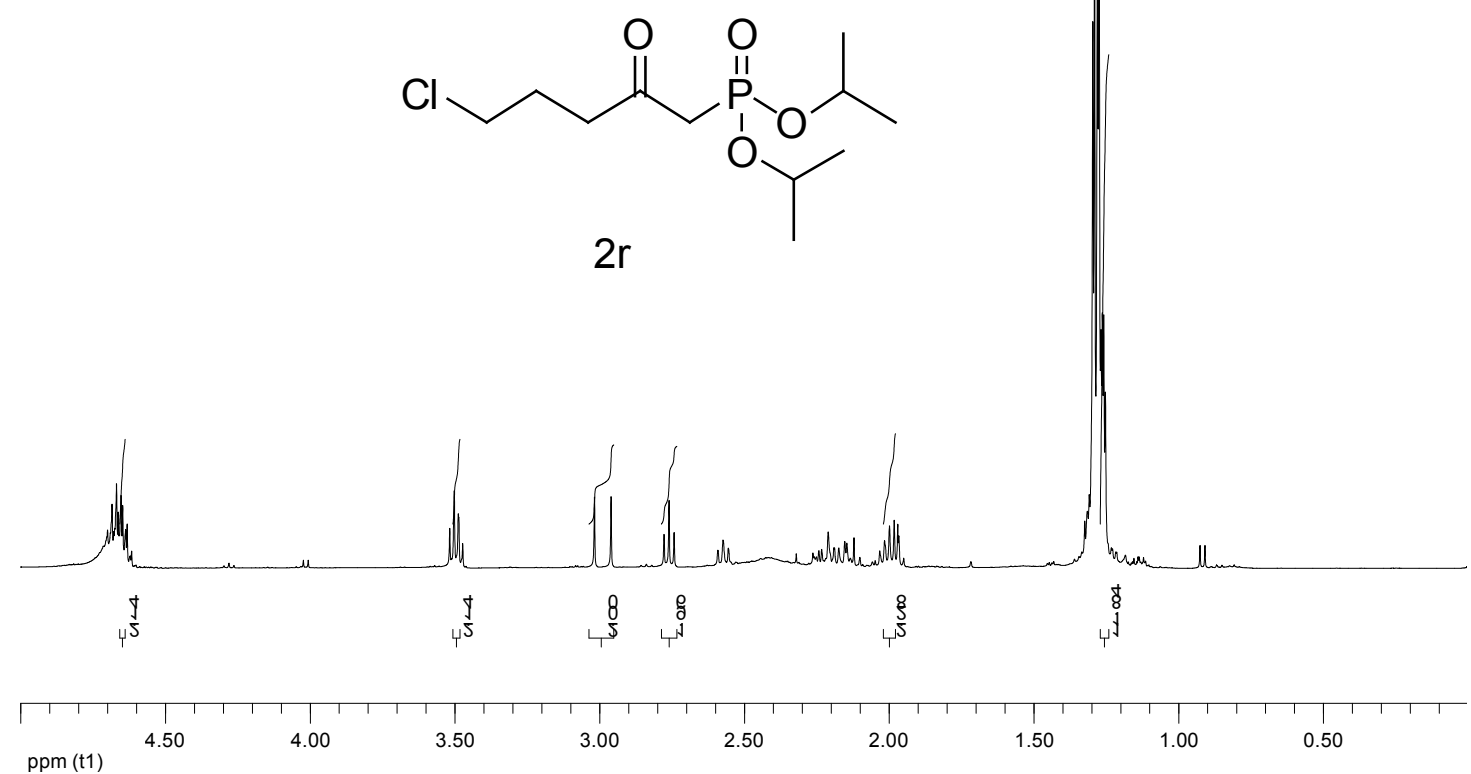




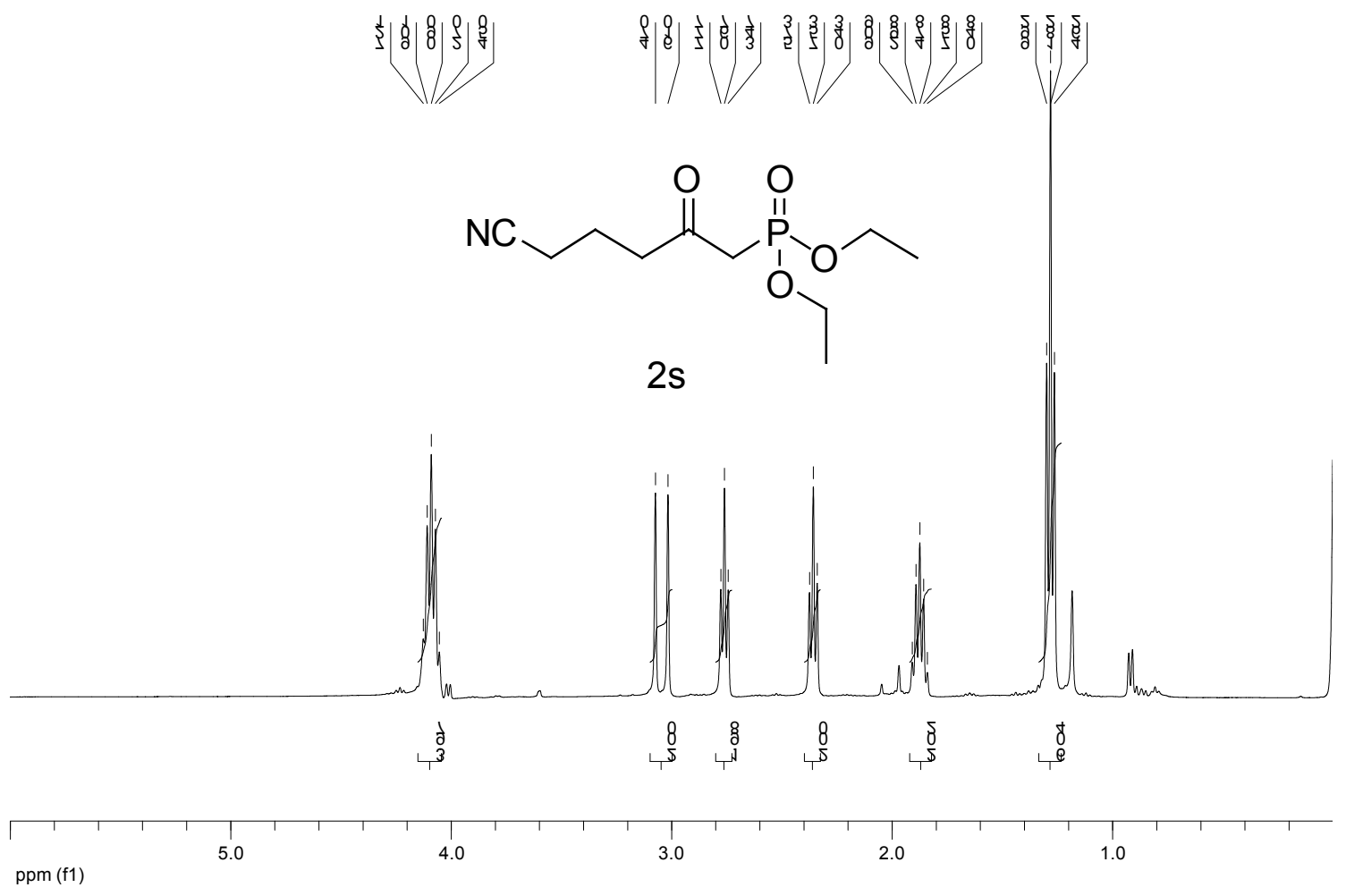

9888 ig<smiles>CCOP(=O)(CC(=O)C1=CCCCC1)OCC</smiles>

$2 t$

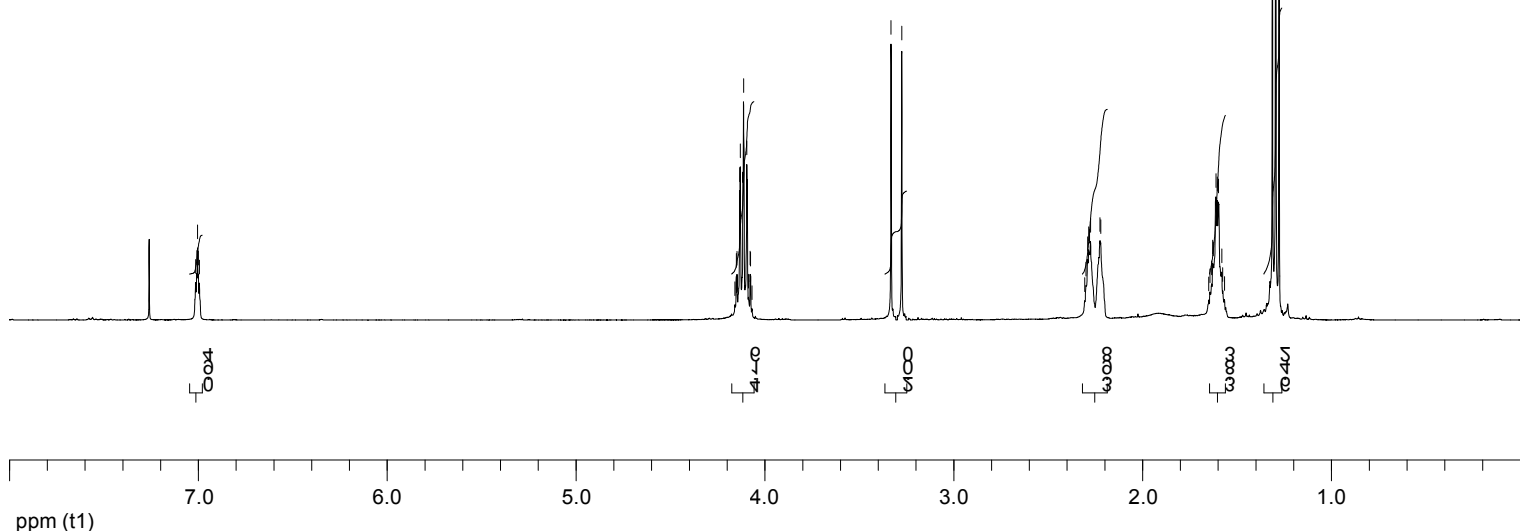




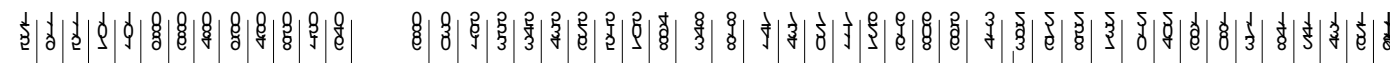<smiles>CCOP(=O)(CC(=O)C1CCCCC1)OCC</smiles>

$2 u$

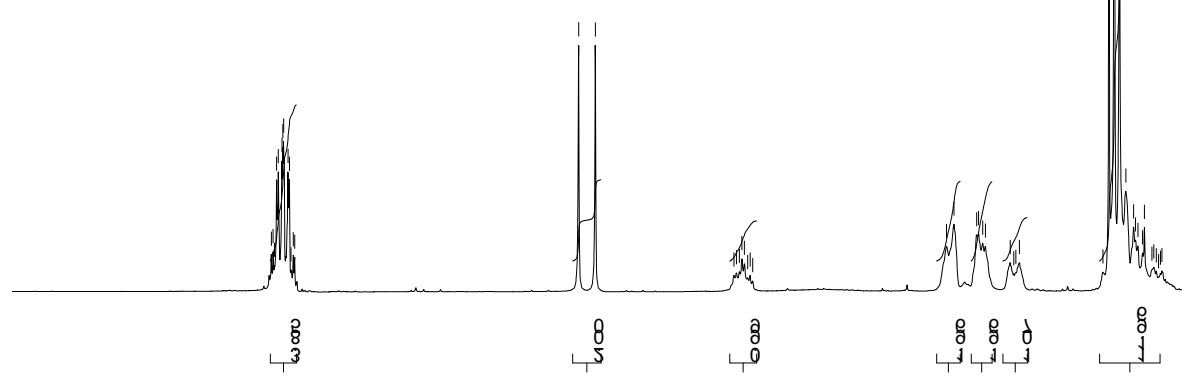

ppm (t1)

4.0

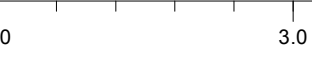

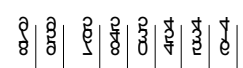

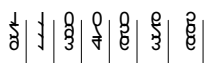

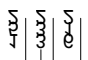<smiles>CCOP(=O)(CC(=O)c1ccccc1)OCC</smiles>

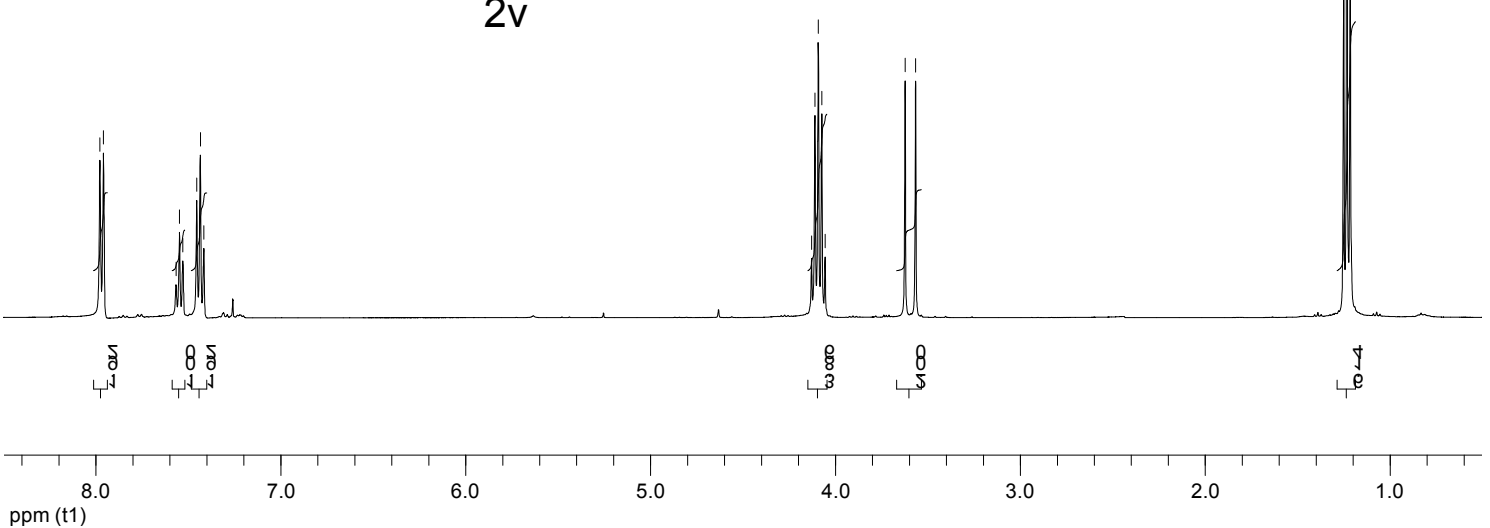




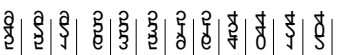

ริ $\mid$ |

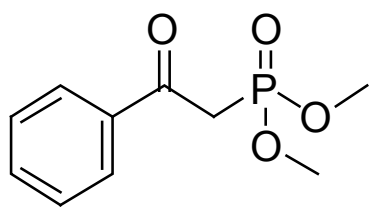

$2 w$
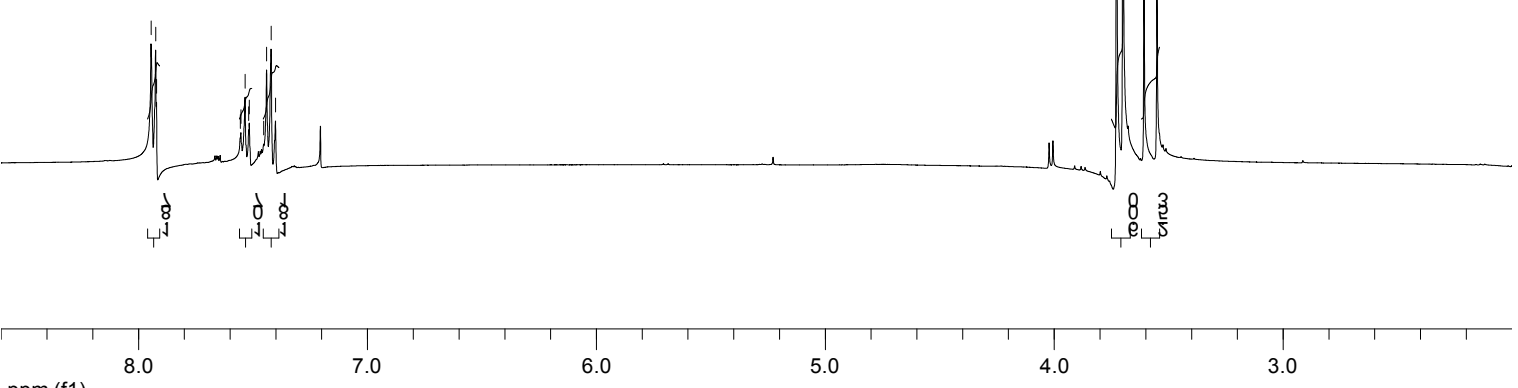

ppm (f1)

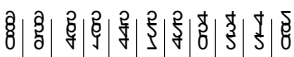

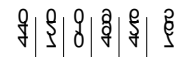

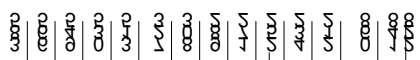<smiles>[X]CCCOP(=O)(CC(=O)c1ccccc1)OCCCC</smiles>

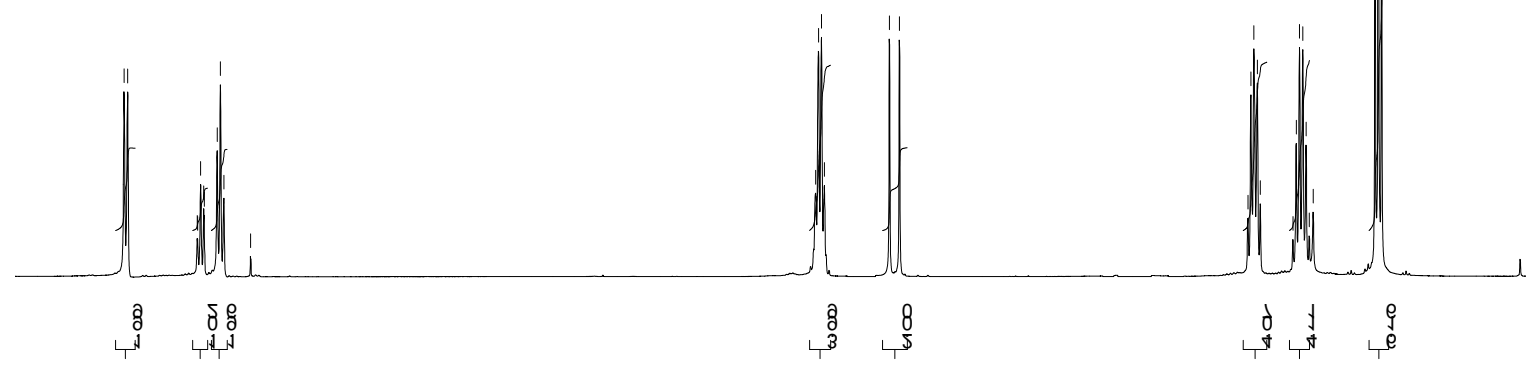

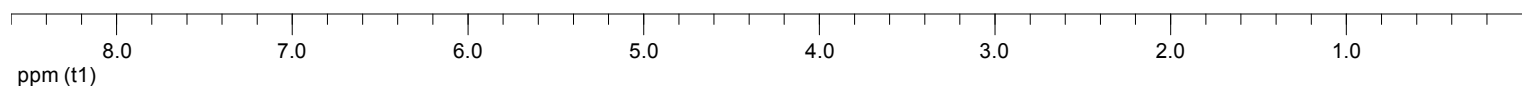




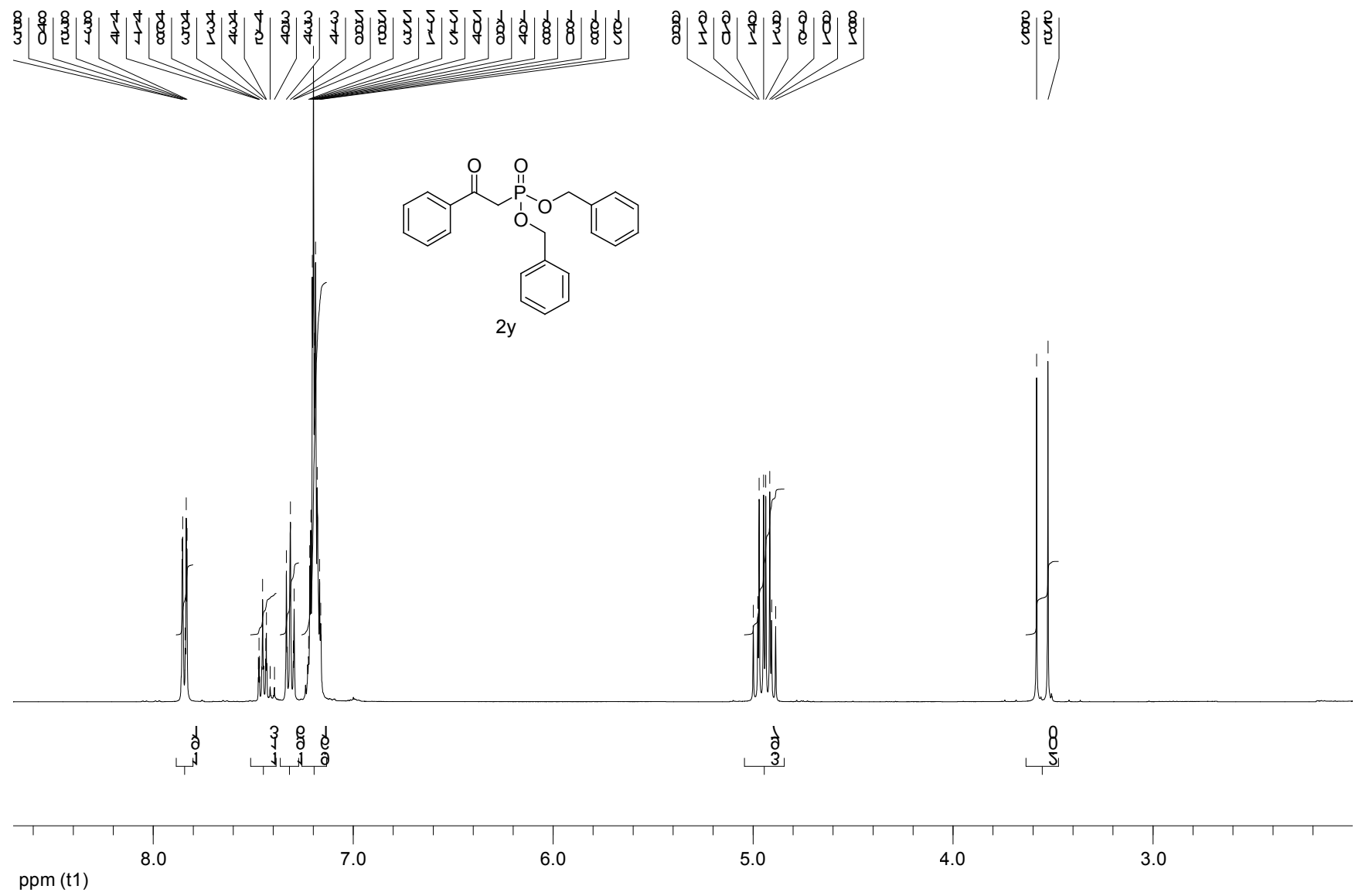


